\title{
Molecular and cellular signatures underlying superior immunity against Bordetella pertussis upon pulmonary vaccination
}

\author{
RHM Raeven ${ }^{1,2,6}$, J Brummelman ${ }^{3,4,6}$, JLA Pennings ${ }^{5}$, L van der Maas ${ }^{1}, \mathrm{~K} \mathrm{Helm}^{3}$, W Tilstra ${ }^{1}$, \\ A van der Ark ${ }^{1}$, A Sloots ${ }^{1}, \mathrm{P}$ van der Ley ${ }^{1}, \mathrm{~W}$ van Eden ${ }^{4}, \mathrm{~W}$ Jiskoot $^{2}, \mathrm{E}$ van Riet ${ }^{1}, \mathrm{CACM}$ van Els ${ }^{3}$, \\ GFA Kersten ${ }^{1,2}$, WGH $\mathrm{Han}^{3,7}$ and B Metz ${ }^{1,7}$
}

Mucosal immunity is often required for protection against respiratory pathogens but the underlying cellular and molecular mechanisms of induction remain poorly understood. Here, systems vaccinology was used to identify immune signatures after pulmonary or subcutaneous immunization of mice with pertussis outer membrane vesicles. Pulmonary immunization led to improved protection, exclusively induced mucosal immunoglobulin A (IgA) and T helper type 17 (Th17) responses, and in addition evoked elevated systemic immunoglobulin G (IgG) antibody levels, IgG-producing plasma cells, memory B cells, and Th17 cells. These adaptive responses were preceded by unique local expression of genes of the innate immune response related to Th17 (e.g., Rorc) and IgA responses (e.g., Pigr) in addition to local and systemic secretion of Th1/Th17-promoting cytokines. This comprehensive systems approach identifies the effect of the administration route on the development of mucosal immunity, its importance in protection against Bordetella pertussis, and reveals potential molecular correlates of vaccine immunity to this reemerging pathogen.

\section{INTRODUCTION}

Optimal protection against respiratory pathogens requires induction of mucosal immunity in the lungs, where tissueresident memory $\mathrm{T}$ cells ${ }^{1,2}$ and immunoglobulin $\mathrm{A}(\operatorname{IgA})$ antibodies $^{3}$ play a pivotal role in the rapid recognition of pathogens. Therefore, induction of these responses by immunization is appealing. Unfortunately, current vaccines against respiratory pathogens, including Bordetella pertussis, which causes whooping cough, do not induce mucosal immunity. Improvement of pertussis vaccines and optimized vaccination strategies $^{4,5}$ are required, as whooping cough persists as an endemic disease even in highly vaccinated populations. Acellular pertussis vaccine induces a systemic immune response characterized by the formation of immunoglobulin $\mathrm{G}$ (IgG) antibodies and a T helper type 2 (Th2)-dominated response, ${ }^{6-9}$ whereas a Th1/Th17 response is preferable for protection against infection. ${ }^{8}$ Subcutaneous immunization of outer membrane vesicle pertussis vaccine (omvPV) could be an improved alternative, as it elicits high serum IgG levels against a broad antigen range ${ }^{10}$ and a mixed Th1, Th17, and Th2 response. ${ }^{11}$ This response is comparable to the response induced by a $B$. pertussis infection, but importantly lacks the mucosal immune responses. ${ }^{8,12-14}$

Vaccine administration directly into the respiratory tract provides better protection, compared with intramuscular administration, because of its induction of local immune responses. ${ }^{15-18}$ Protection against $B$. pertussis challenge has been achieved with various intranasal pertussis vaccine candidates. ${ }^{19-22}$ However, direct comparison of local and systemic immune responses induced by subcutaneous vs. mucosal immunization has not been performed.

${ }^{1}$ Institute for Translational Vaccinology (Intravacc), Bilthoven, The Netherlands. ${ }^{2}$ Division of Drug Delivery Technology, Cluster BioTherapeutics, Leiden Academic Centre for Drug Research, Leiden, The Netherlands. ${ }^{3}$ Centre for Infectious Disease Control, National Institute for Public Health and the Environment, Bilthoven, The Netherlands. ${ }^{4}$ Department of Infectious Diseases and Immunology, Utrecht University, Utrecht, The Netherlands and ${ }^{5}$ Centre for Health Protection, National Institute for Public Health and the Environment, Bilthoven, The Netherlands. Correspondence: RHM Raeven (Rene.raeven@intravacc.nl)

${ }^{6}$ These authors contributed equally to this work.

${ }^{7}$ These authors contributed equally to this work 
In this study, we used systems vaccinology, a valuable tool to unravel molecular signatures and predict vaccine responsiveness, ${ }^{23-26}$ to investigate in detail how the route of immunization, i.e., pulmonary vs. subcutaneous, of a pertussis vaccine candidate affects protection and the quality of immune responses in mice (Figure 1a). As pertussis vaccine candidate we used an omvPV prepared from a genetically modified $B$. pertussis B1917 strain with retained pertactin (Prn), an immunogenic virulence factor that is normally cleaved from the bacterial cell surface. ${ }^{27}$ The B. pertussis B1917 strain is phylogenetically relevant to the current circulating strains that may be responsible for the pertussis resurgence, ${ }^{28}$ whereas the enhanced presence of Prn may favor the detectability of vaccine antigen-specific cellular immune responses. Besides $B$. pertussis clearance from the respiratory tract after intranasal challenge, we determined gene expression profiles in draining lymph nodes and lungs, and cytokine profiles and antibody responses in serum and lungs of immunized mice. Finally, specific B-cell and $\mathrm{T}$-cell responses were investigated both locally and systemically. Our results demonstrate innate and adaptive

a

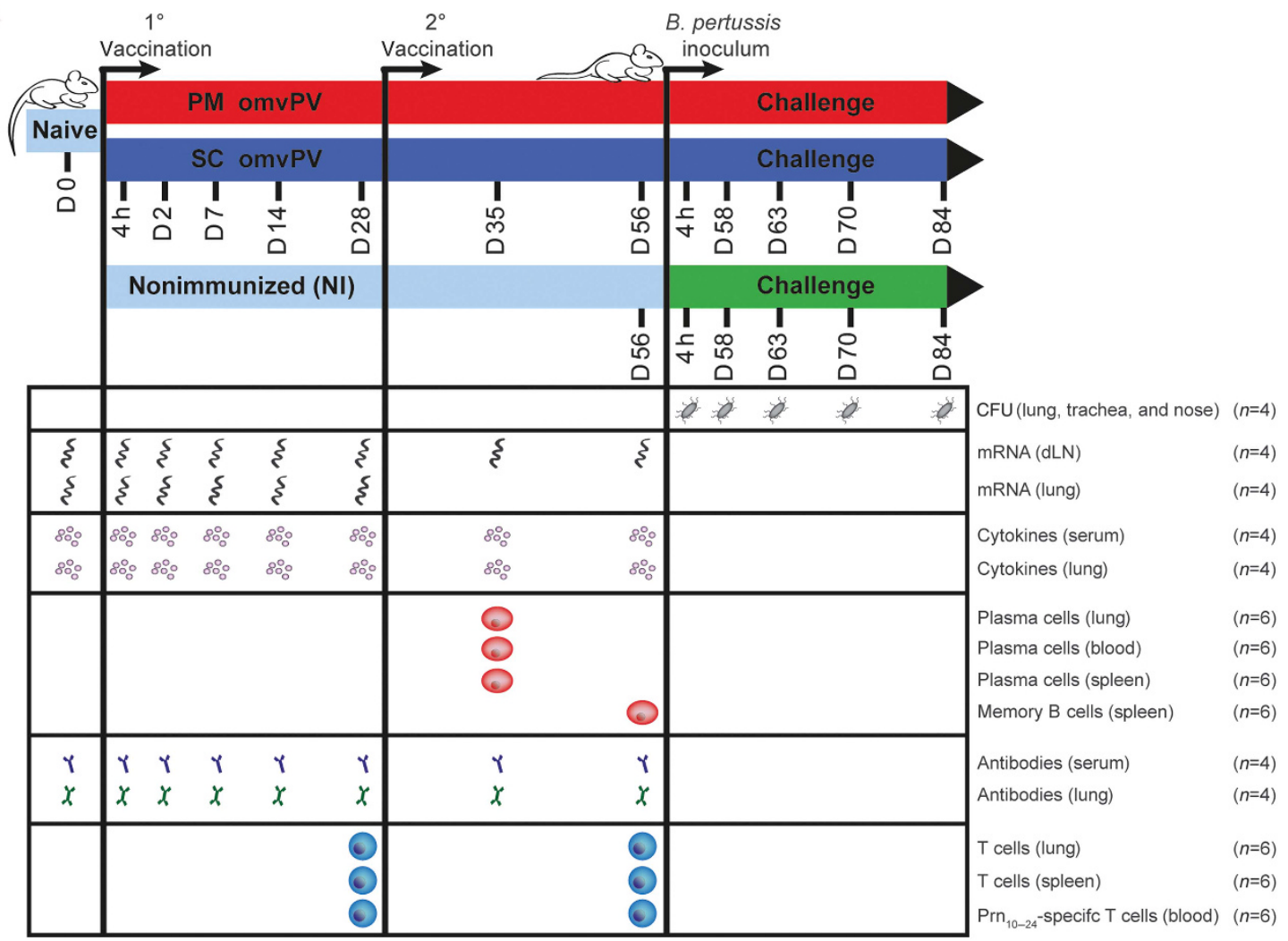

b

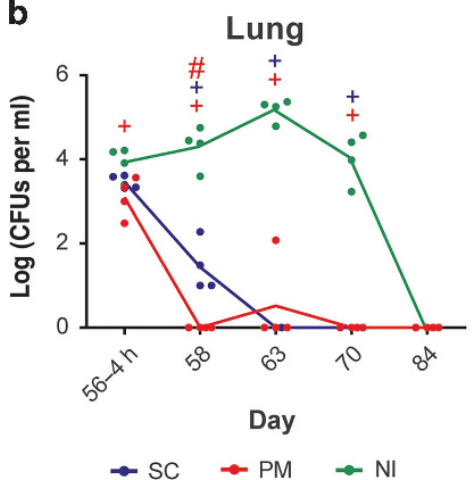

C

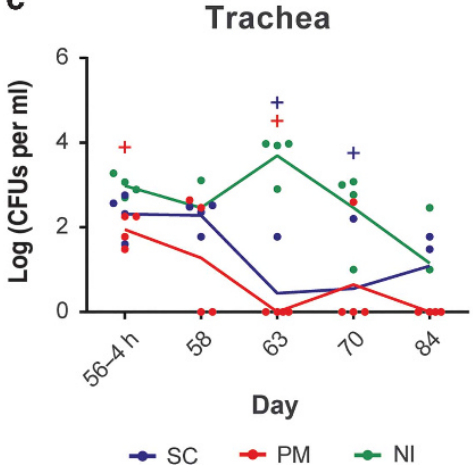

d

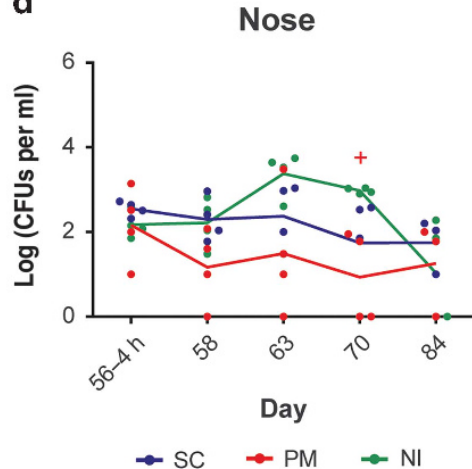

Figure 1 Study design and Bordetella pertussis colonization measured in the respiratory tract. (a) BALB/c mice were immunized with $1 \mu \mathrm{g}$ outer membrane vesicle pertussis vaccine (omvPV) by the pulmonary (PM; red) or subcutaneous (SC; blue) route on days 0 and 28 . Subsequently, for both routes the vaccination-induced responses were characterized over a period of 56 days at 7 time points. In addition, a $B$. pertussis challenge $\left(2 \times 10^{5}\right.$ colony-forming units (CFUs)) was performed on day 56 in both vaccinated groups and nonimmunized (NI) mice (green). Vaccination responses were characterized at the transcriptomic, proteomic, and cellular levels on given time points, as depicted. (b-d) The number of CFUs were determined after $B$. pertussis challenge in (b) lungs, (c) trachea, and (d) nose lavages of SC, PM, and NI mice. ${ }^{\#} P<0.05$ vs. SC mice; ${ }^{+} P<0.05$ vs. $\mathrm{NI}$ mice. 
hallmarks of superior protective immunity to $B$. pertussis as conferred by pulmonary vaccination with omvPV.

\section{RESULTS}

Superior protection against $B$. pertussis infection by pulmonary compared with subcutaneous omvPV immunization

Lungs of nonimmunized control mice (NI mice) were heavily colonized after a $B$. pertussis challenge with highest numbers of colony-forming units found 6 days after challenge (Supplementary Figure S1 online). A dose-dependent immunization study comparing 1 and $10 \mu \mathrm{g}$ omvPV demonstrated that administration of $10 \mu \mathrm{g}$ omvPV resulted in complete lung clearance of pulmonary immunized mice (PM mice) and most of subcutaneously immunized mice (SC mice) 3 days after challenge. (Supplementary Figure S1). Immunization with $1 \mu \mathrm{g}$ revealed that lungs of PM mice were cleared 4 days after challenge, whereas SC mice were colonized 3 days longer. Based on these results we decided to investigate the immunization with $1 \mu \mathrm{g}$ omvPV for both routes in more detail in a follow-up study (Figure 1a). The lungs, and also trachea and noses, were again heavily colonized in NI mice (Figure 1b-d). In contrast, B. pertussis was again mostly cleared from lungs of PM mice just 2 days after challenge, whereas those of SC mice were cleared 5 days later (Figure 1b). In the trachea of both SC and PM mice, bacteria were mostly cleared 7 days after challenge (Figure 1c). In the nose, no complete clearance of $B$. pertussis was observed in all groups within 28 days after challenge. However, the number of bacteria in noses of PM mice was significantly lower than in NI mice 14 days after challenge (Figure 1d).

\section{Improved humoral responses after PM immunization}

Both immunization routes induced high levels of anti-OMV IgM and IgG antibodies in serum, yet with significantly higher levels after PM immunization on day 56 (Figure 2a,b). In both groups, the primary immunization was dominated by IgG1, whereas the booster immunization showed an increased contribution of IgG3 (Figure 2c). PM immunization elicited anti-Prn IgG antibodies only after booster immunization, whereas they were already observed after primary SC immunization (Supplementary Figure S2a). Moreover, booster PM immunization led to increased values of anti-Prn IgG2a and IgG2b (Supplementary Figure S2b).

The specificity of anti-OMV IgG antibodies was identified (Supplementary Figure S3a-c) and revealed antibodies against 12 immunogenic B. pertussis proteins after PM and 17 after SC immunization (Supplementary Figure S3a,b and Supplementary Table S1). Of note, this method detects linear but not conformational epitopes. Nevertheless, the results indicated a distinct repertoire of antibody specificities depending on the route of vaccine administration. Spots showing high staining intensities in both groups corresponded with proteins BP3755, Vag8, BrkA, and two unidentified proteins U1-2. Antibodies against AhcY and OmpA(2) were induced solely by PM immunization, whereas antibody formation against $\mathrm{OmpA}(1), \mathrm{BfrG}$, GroEl, PurH, and two unidentified proteins (U3-4) were induced solely by SC immunization. In addition, anti-lipopolysaccharide antibodies $(10 \mathrm{kDa})$ were produced after booster immunization in both groups (Supplementary Figure S3c). This antilipopolysaccharide response seems lower compared with the response described previously with wild-type B1917 omvPV, ${ }^{10,11}$ most likely because of a lower immunization dose. Notably, no IgG antibodies could be detected against the antigens present in acellular pertussis vaccines (Ptx, FHA, and Fim2/3) except for anti-Prn IgG after omvPV immunization via either route (data not shown).

IgA antibodies were found exclusively in PM mice in both serum (Figure 2d) and lungs (Figure 2e). These IgA antibodies were directed against OMV and Prn (Figure 2d and Supplementary Figure S2c,d) but not against other antigens present in acellular vaccines (data not shown). Anti-OMV IgA in the lung was mainly directed against Vag8 and lipopolysaccharide (Figure 2e and Supplementary Figure S3a,d).

At the peak of the plasma cell response (day 35), similar and modest numbers of anti-OMV and anti-Prn IgG-secreting cells were found in blood and spleen of PM and SC mice (Figure $2 \mathbf{f}$ and Supplementary Figure S2e). They were also detected in the lungs of PM mice, but not of SC mice. In addition, anti-OMV and anti-Prn IgA-secreting cells were detected only in the lungs, spleen, and blood of PM mice (Figure $\mathbf{2 f}$ and Supplementary Figure S2e). With respect to IgG-producing memory B cells, no significant difference was detected in spleens of PM or SC mice (day 56), although a trend was observed toward higher numbers in the PM mice (Figure 2g and Supplementary Figure S2f). Moreover, no anti-OMV and anti-Prn IgA-producing memory $\mathrm{B}$ cells could be detected in all mice.

Overall, the data show that PM immunization induced an improved B-cell response compared with SC immunization indicated by increased serum IgM and IgG levels, presence of pulmonary IgA antibodies, induction of pulmonary IgGproducing plasma cells, and both systemic and pulmonary IgAproducing plasma cells.

\section{Enhanced quantity and quality of $\mathrm{CD} 4{ }^{+} \mathrm{T}$-cell responses by PM immunization}

The magnitude of $\mathrm{CD} 4{ }^{+} \mathrm{T}$-cell responses was investigated by determining the total percentage of $B$. pertussis-specific cytokine-producing (interferon- $\gamma$ (IFN $\gamma$ ), interleukin (IL)-5, or IL-17A) $\mathrm{CD}^{+} \mathrm{T}$ cells using intracellular cytokine staining (ICS) analysis. Both PM and SC immunization induced a significant increase in OMV-specific and Prn-specific cytokineproducing $\mathrm{CD} 4{ }^{+} \mathrm{CD} 44^{+} \mathrm{T}$ cells already on day 28 (Figure 3a and Supplementary Figure S4a). On day 56, a significantly higher percentage of these cells were detected after PM compared with SC immunization. This difference was also observed ex vivo at the epitope level, as $\operatorname{Prn}_{10-24}$-specific CD4 ${ }^{+}$ $\mathrm{T}$ cells were exclusively detected in the blood of PM mice (Figure 3b). 
a

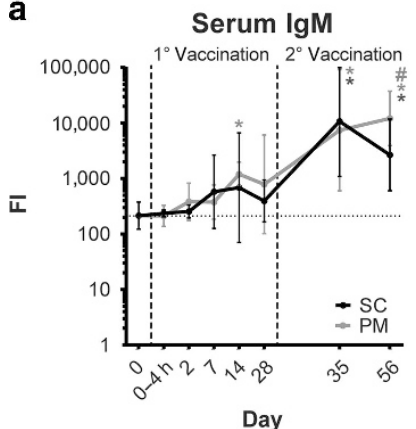

d

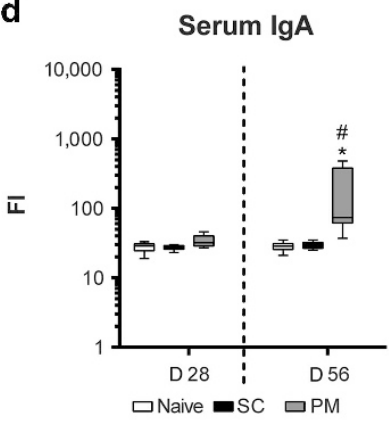

f

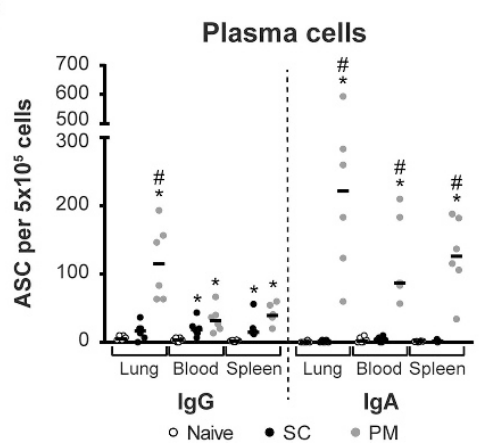

b
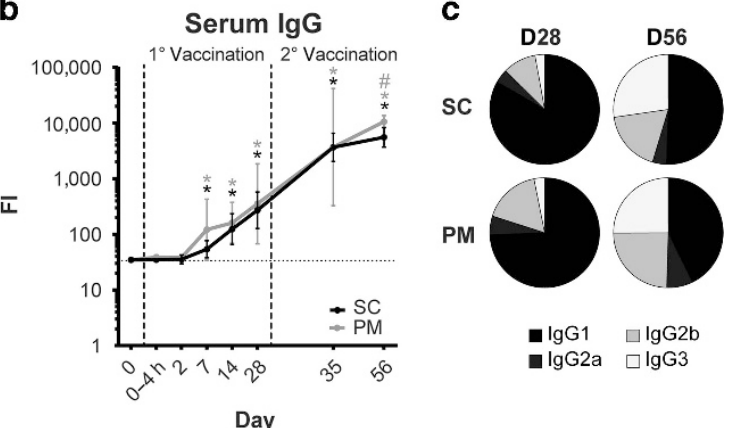

e

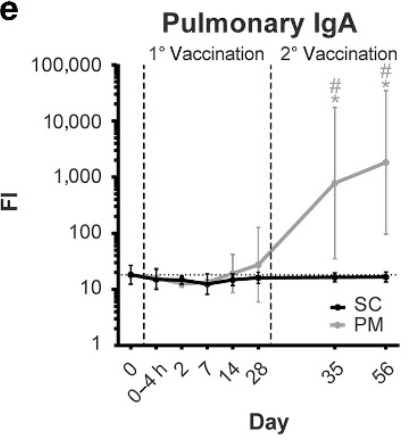

g

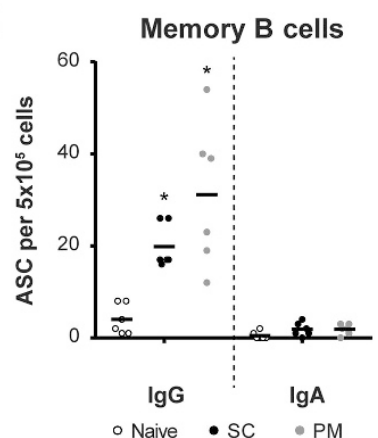

Figure 2 Bordetella pertussis outer membrane vesicle (OMV)-specific B-cell responses induced by pulmonary (PM) and subcutaneous (SC) immunization with omvPV. (a-e) Anti-OMV (a) immunoglobulin M (IgM), (b) immunoglobulin G (IgG) antibody levels and (c) IgG subclass distribution were determined in sera. Anti-OMV immunoglobulin A ( $\lg A)$ antibody levels were determined in (d) sera and (e) lung lysates. Results are expressed as fluorescence intensities (FI) of 4 mice per group per time point. ${ }^{*} P<0.05$ vs. naive mice (day 0 ); ${ }^{\#} P<0.05$ vs. SC mice. (f, $\mathbf{g}$ ) Numbers of (f) OMV-specific IgG- and IgA-secreting plasma cells in lungs, blood, and spleens and (g) numbers of IgG-and IgA-producing memory cells in spleens were determined by B-cell ELISpot of 6 mice per group at day 35 and day 56 , respectively. Results are indicated as antibody-secreting cells (ASCs) per $5 \times 10^{5}$ cells. ${ }^{\star} P<0.05$ vs. naive mice; ${ }^{\#} P<0.05$ vs. SC mice.

Cytokine levels in the culture supernatants of OMVstimulated splenocytes revealed that lower levels of Th2 cytokines (IL-4 and IL-5) were secreted by cells from PM immunized mice as compared with SC immunization (Supplementary Figure S5a,b). In contrast, increased production of IL-13 was found (Supplementary Figure S5b). Moreover, only PM immunization induced specific IFN $\gamma$ production and caused higher production of IL-17 and IL-10 (Supplementary Figure S5b). ICS analysis showed a significantly increased percentage of OMV-specific IFN $\gamma$ producing $\mathrm{CD} 4{ }^{+} \mathrm{CD} 44^{+} \mathrm{T}$ cells after $\mathrm{PM}$ immunization on day 28 but not at day 56 (Figure 3c). OMV-specific IL-5producing $\mathrm{CD} 4{ }^{+} \mathrm{CD} 44^{+}$T cells were detectable in both PM and SC mice on days 28 and 56, but were significantly higher in the SC mice (Figure 3d). OMV-specific IL-17A-producing $\mathrm{CD} 4{ }^{+} \mathrm{CD} 44^{+} \mathrm{T}$ cells were induced after SC immunization on day 56. Markedly, significantly higher percentages of these cells were detected after PM immunization at both days 28 and 56 (Figure 3e). A similar trend for Prn-specific CD $4^{+} \mathrm{CD} 44^{+}$ $\mathrm{T}$ cells was observed (Supplementary Figure S4b-d). The distribution of OMV-specific and Prn-specific CD ${ }^{+} \mathrm{CD} 44^{+}$ $\mathrm{T}$ cells, based on the production of IFN $\gamma$, IL-5, or IL-17A, indicates that SC immunization induced a Th1-dominated systemic response on day 28 and a mixed Th1/Th17/Th2 response on day 56 (Figure $3 \mathrm{f}$ and Supplementary Figure S4e). In contrast, PM immunization induced a mixed Th1/Th17 
a

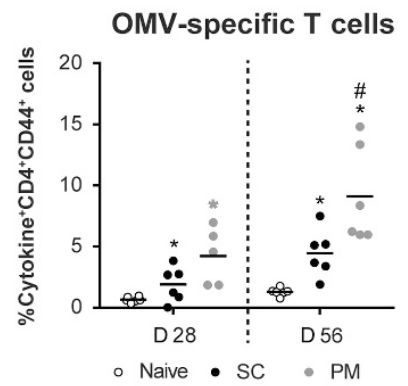

d

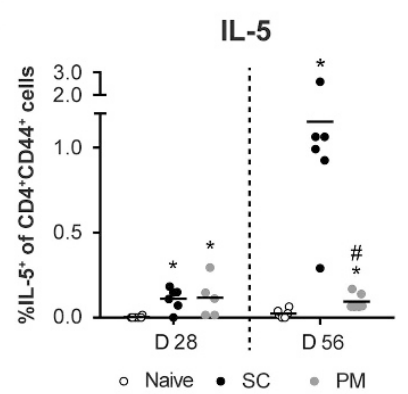

g

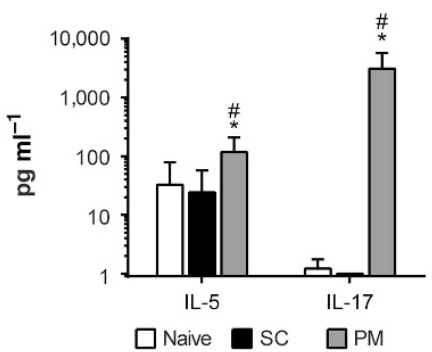

b

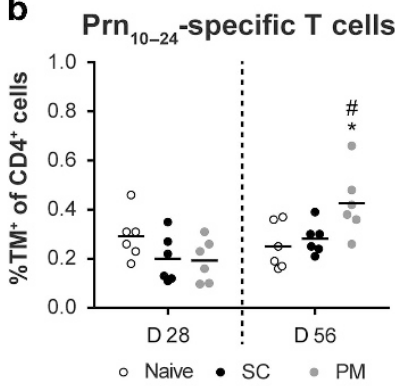

e

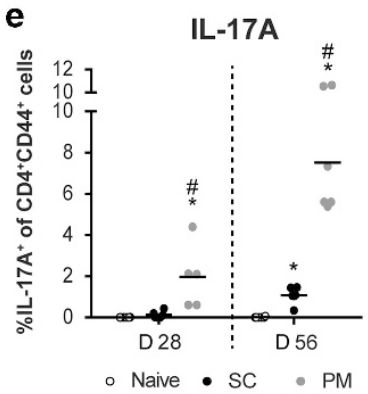

h

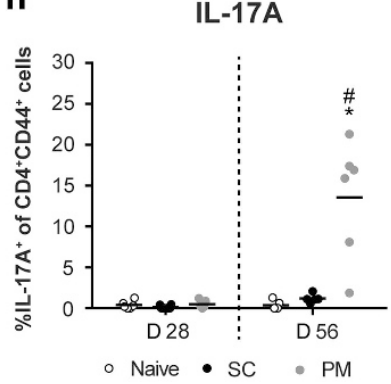

C

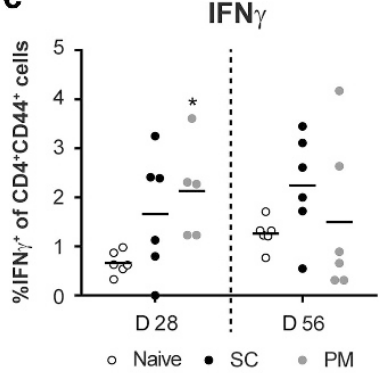

f

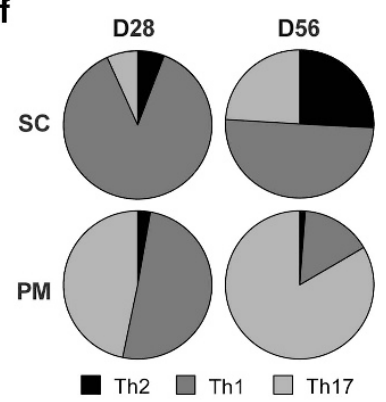

i

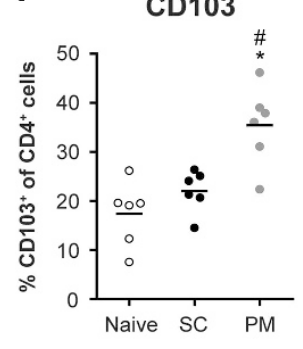

Figure 3 Pulmonary and systemic Bordetella pertussis outer membrane vesicle (OMV)-specific T-cell responses induced by pulmonary

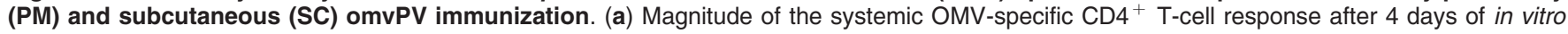
stimulation with OMV was determined using intracellular cytokine staining (ICS) on splenocytes, calculated as the total percentage of cytokine (interleukin (IL)-5, interferon- $\gamma$ (IFN $\gamma$ ), and IL-17A)-producing CD4 ${ }^{+} \mathrm{CD}_{4} 4^{+}$T cells. (b) Frequency of $\mathrm{Prn}_{10-24}$-specific CD4 ${ }^{+} \mathrm{T}^{-}$cells in blood was determined directly ex vivo on days 28 and 56 (D28 and D56) using a tetramer staining and flow cytometry. (c-e) Percentage of (c) IFN $\gamma-$, (d) IL-5-, and (e) IL-17-producing $\mathrm{CD} 4^{+} \mathrm{CD} 44^{+} \mathrm{T}$ cells of spleens harvested on days 38 and 56 and stimulated in vitro for 4 days with OMVs. Results of each analysis are given for 6 mice per group. (f) Distribution of T helper (Th) subsets based on IL-5 (Th2), IFN $\gamma$ (Th1), and IL-17A (Th17) production, as determined by ICS and flow cytometry. ${ }^{*} P<0.05$ vs. naive mice; ${ }^{\#} P<0.05$ vs. SC mice. As determined by multiplex immunoassay (MIA), (g) IL-5 and IL-17A levels in $3-d a y$ culture supernatant of lung cells, isolated on day 56, after in vitro stimulation with OMVs. Results in $\mathrm{pg} \mathrm{ml}^{-1}$ are corrected for the background level (IMDM complete medium control) and are given as mean \pm s.d. of 6 mice per group. (h) Percentage of IL-17-producing CD4 ${ }^{+} \mathrm{CD} 44^{+} \mathrm{T}$ cells in the lungs, harvested on days 28 and 56, as measured by ICS and flow cytometry after 4 days of in vitro stimulation with OMVs. (i) Flow cytometry for expression of CD103 on $\mathrm{CD}^{+}{ }^{+} \mathrm{CD} 44^{+}$T cells in the lungs on day 56.

response on day 28 that shifted toward a Th17-dominated response on day 56.

With respect to mucosal immune responses, OMV-stimulated lung cells of PM mice revealed moderate IL-5 and vigorous IL-17A secretion in supernatant (Figure 3g). In line with this, OMVspecific IL-17A-producing CD4 ${ }^{+} \mathrm{CD} 44^{+} \mathrm{T}$ cells were solely detected in PM mice (Figure 3h). Expression of CD103, a marker for tissue-resident memory $T$ cells, was found on $57 \pm 24 \%$ of these $\mathrm{T}$ cells (data not shown). Moreover, an increased percentage of pulmonary $\mathrm{CD}^{+}{ }^{+} \mathrm{CD} 103^{+} \mathrm{T}$ cells were detected in PM mice compared with SC mice and naive mice (Figure 3i).

In conclusion, PM immunization elicits more systemic Th1/ Th17-skewed CD4 ${ }^{+} \mathrm{T}$ cells as well as more tissue-resident Th17 $\mathrm{CD}^{+}{ }^{+} \mathrm{T}$ cells compared with SC immunization.
Early cytokine signatures suggest increased Th1 and Th17 responses after PM immunization

Largest differences in cytokine levels were observed $4 \mathrm{~h}$ after primary immunization in serum and lung lysates (Figures 4 and 5). In serum, increased levels of Th1-associated cytokines CCL4 and CXCL10 were found in both groups, with higher levels of CXCL10 in SC mice, whereas CCL5 was observed only in SC mice (Figure 4). IL-5, related to a Th2 response, and CCL2, related to Th2 and Th17 responses, were observed only in SC mice. Other Th17-associated cytokines (CXCL1, granulocyte-colony stimulating factor (G-CSF), IL-6, and tumor necrosis factor- $\alpha(\mathrm{TNF} \alpha)$ ) were increased in both groups, with G-CSF higher in SC mice and IL-6 higher in PM mice. In addition, IL-10 was enhanced in both groups. 


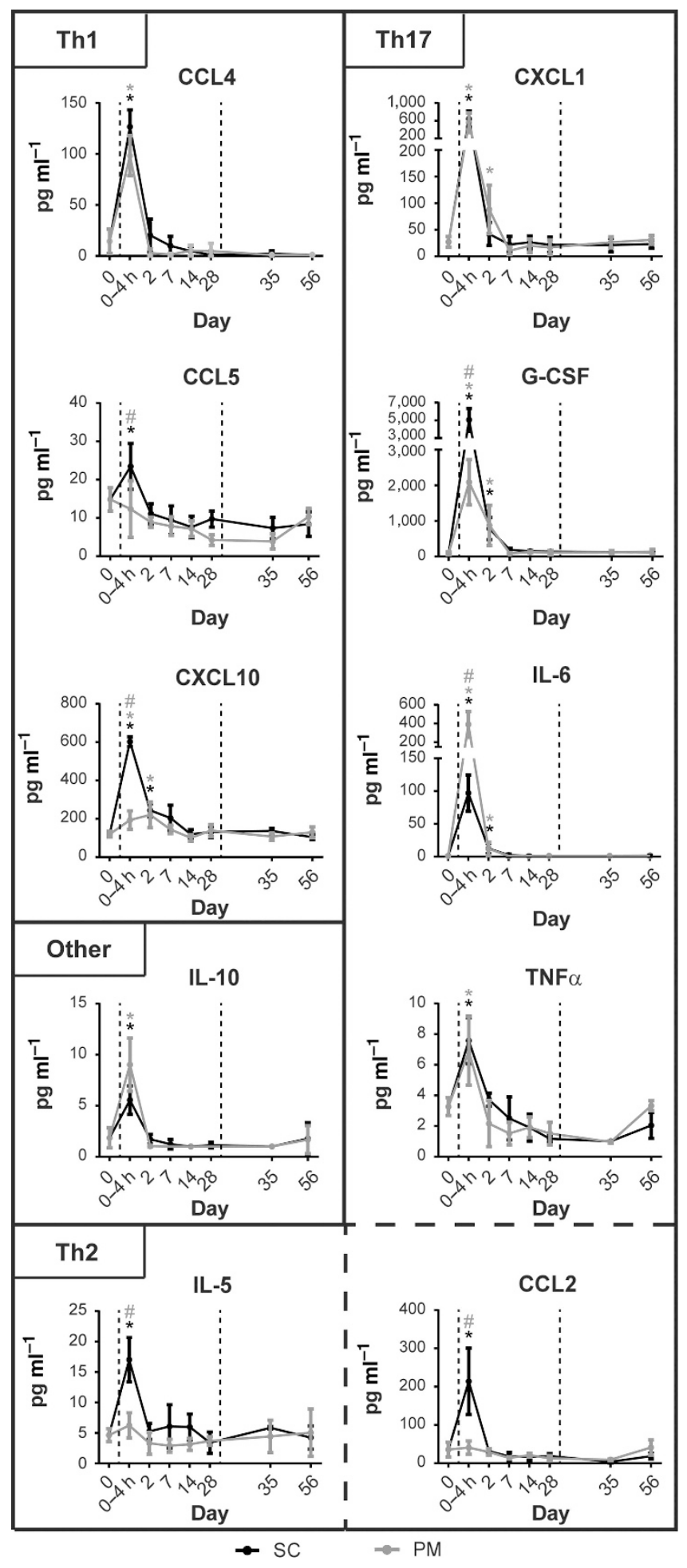

Figure 4 Systemic cytokine responses after pulmonary (PM) and subcutaneous (SC) outer membrane vesicle pertussis vaccine (omvPV) immunization. The concentrations of cytokines in serum of immunized mice were analyzed over time by using multiplex immunoassay (MIA). Cytokines are grouped based on their association with a Th1, Th2, or Th17 response. Data are given as mean concentrations for 4 mice per group per time point. ${ }^{*} P<0.05$ vs. naive mice (day 0 ); ${ }^{\#} P<0.05$ vs. SC mice.
In lung lysates, cytokines associated with a Th1 response (CCL3, CCL4, CXCL9, CXCL10, IL-12p70), Th2 response (CCL2, IL-13), and Th17 response (CCL2, CXCL1, CXCL2, G-CSF, GM-CSF, IL-1 $\beta$, IL-6, IL-17A, TNF $\alpha$ ) as well as CXCL5, IL-1 $\alpha$, IL-15, LIF, and M-CSF were increased in PM mice (Figure 5).

\section{Transcriptomic signatures in draining lymph nodes}

Microarray analysis on draining lymph nodes (dLNs) from PM and SC mice, revealed 1,921 differentially expressed genes (DEGs; $p \leq 0.001$, fold ratio (FR) $\geq 1.5$ ) compared with naive mice (Figure 6a). Of these, 951 were upregulated and 466 downregulated exclusively after PM immunization; 109 were upregulated and 39 were downregulated exclusively after SC immunization; and 211 were upregulated and 145 were downregulated in both PM and SC mice. Overrepresentation analysis (ORA) indicated enrichment of 141 GO-BP (Gene Ontology Biological Process) terms and KEGG (Kyoto Encyclopedia of Genes and Genomes) pathways. A selection of immunologically relevant terms is shown in Figure 6b.

Genes related to T cells and B cells, based on gene ontology and text mining, are shown in Figure $\mathbf{6 c}$, d. Many of the $50 \mathrm{~T}$ cell-related genes were associated with distinct Th subsets (Figure 6c). Upregulation of most genes occurred $4 \mathrm{~h}$ after immunization and from day 7 onwards. More genes were upregulated in PM mice than in SC mice, including Th17associated genes, such as the master regulator for Th17 differentiation (Rorc), Hifla, and Havcrl. The few T cellrelated genes exclusively expressed in SC mice comprised Th1and Th17-associated genes, such as Irf5, Ccr6, and Syt11.

Expression of B cell-related genes in SC mice occurred mainly on days 7 and 35 (Figure 6d). In contrast, elevated expression of $B$ cell-related genes occurred earlier in PM mice ( $4 \mathrm{~h}$ after primary immunization) and persisted until day 28. A smaller number of genes were induced on day 35 by booster immunization in PM compared with SC mice. Expression of $M z b 1$, Ighm, Igkc, Ighg2b, Jchain, Ighg1, and Aicda suggested antibody production and presence of $\mathrm{B}$ cells in dLN that was more pronounced in PM mice. In addition, upregulation of specific B-cell membrane, activation, and homing markers were observed. Both immunization routes induced $C x c r 5, C d 22, C d 40$, and $C d 83$ expression, whereas only the PM route induced $C d 38$, and only the SC route induced $C d 19$, Cd72, Ccr6, and Siglecg.

Many DEGs were found $4 \mathrm{~h}$ after PM immunization. They were mainly involved in cell cycle (data not shown). After SC immunization, in contrast, only $5 \%$ of genes were differentially expressed after $4 \mathrm{~h}$. Notably, in SC mice $72.5 \%$ of the genes upregulated after $4 \mathrm{~h}$ were regulated by interferons as compared with $26 \%$ in PM mice (Supplementary Figure S6a,b). Moreover, $50 \%$ of these genes in SC mice were regulated by all three interferons (IFN $\alpha$, IFN $\beta$, and IFN $\gamma$ ), whereas the majority in PM mice were selectively regulated by only one of the three (Figure 6e and Supplementary Figure S6a,b). 


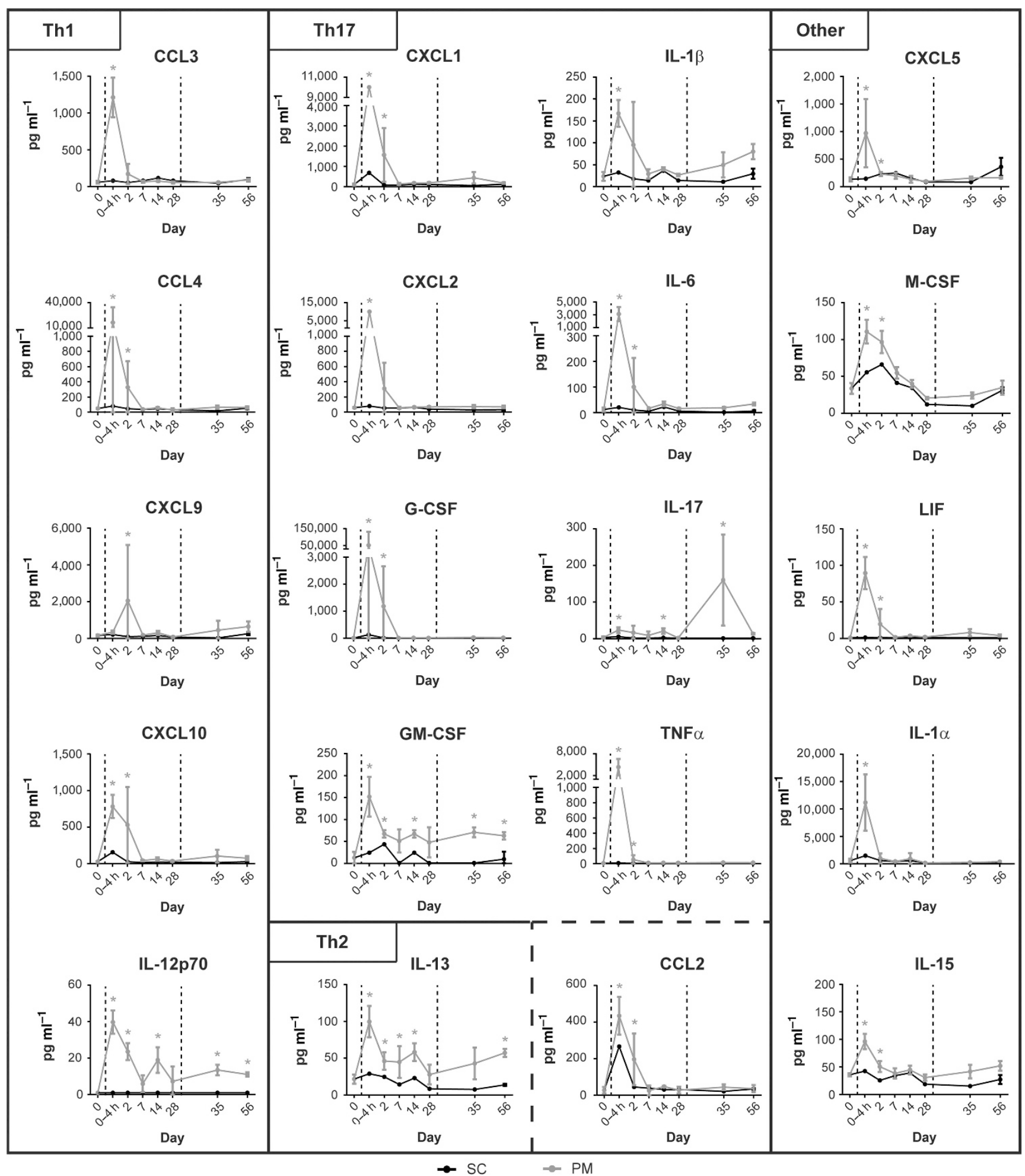

Figure 5 Pulmonary cytokine responses after pulmonary (PM) and subcutaneous (SC) outer membrane vesicle pertussis vaccine (omvPV) immunization. The concentrations of cytokines in lung lysates of immunized mice were analyzed over time by using multiplex immunoassay (MIA). Cytokines are grouped based on their association with a Th1, Th2, or Th17 response. The cytokine concentrations were measured in the individual lung lysates of 4 mice per time point for PM mice and in pooled lung lysates of 4 mice per time point for SC mice. ${ }^{\star} P<0.05$ vs. naive mice (day 0 ).

In conclusion, PM and SC immunization with omvPV evoke distinct innate and adaptive responses in $\mathrm{dLN}$, as detected on transcriptome level.

\section{Pulmonary transcriptomic signatures}

Microarray analysis performed on lung tissue collected during the primary immunization revealed 691 DEGs $(p \leq 0.01$, FR 
a

PM

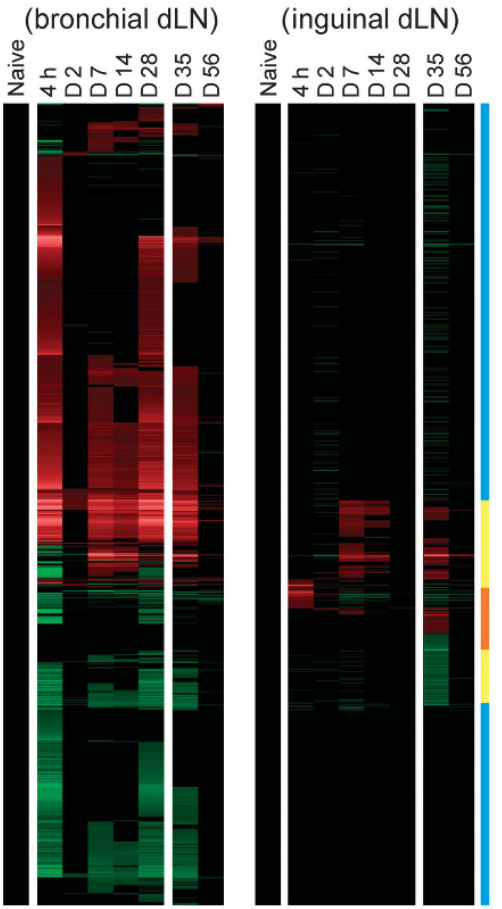

C
SC

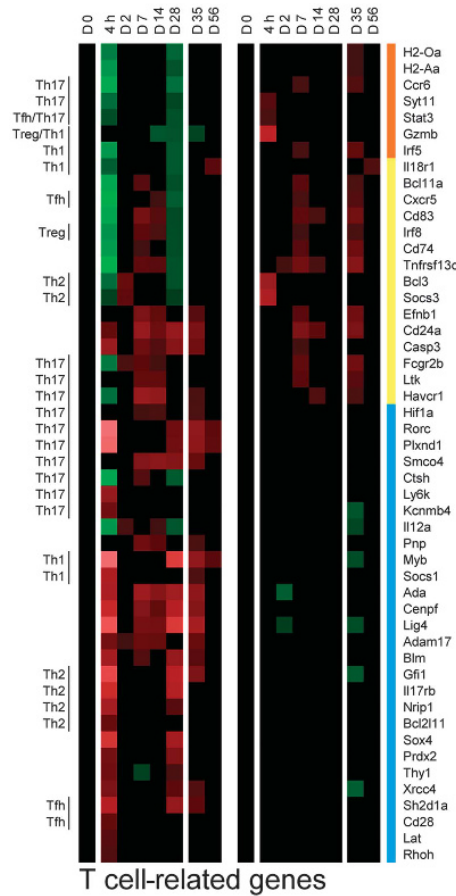

b

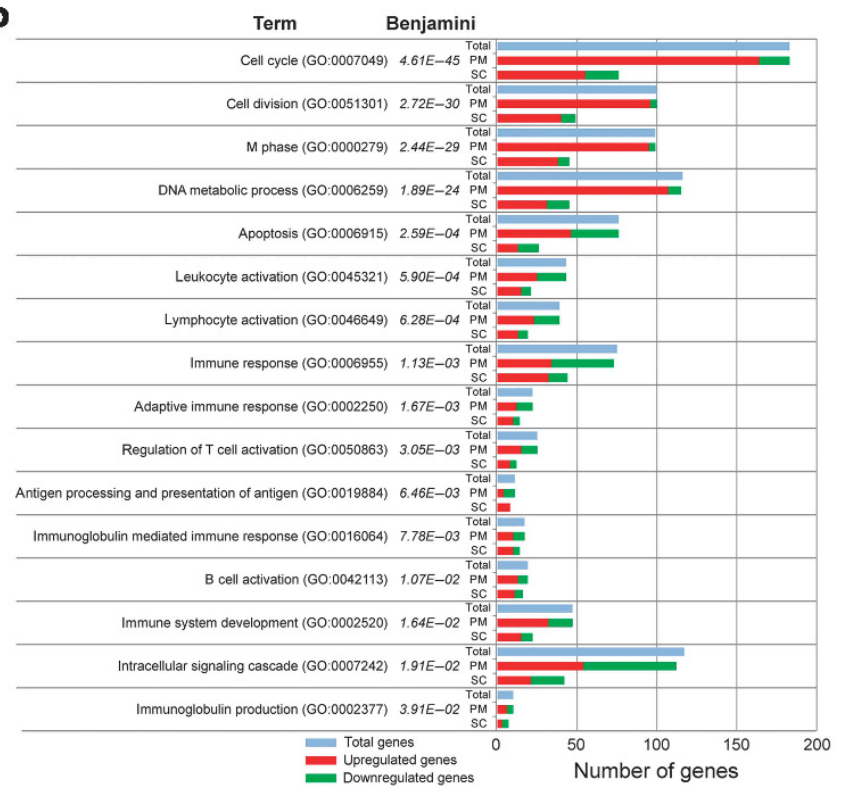

d PM

SC

e

e PM

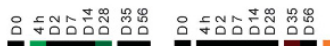
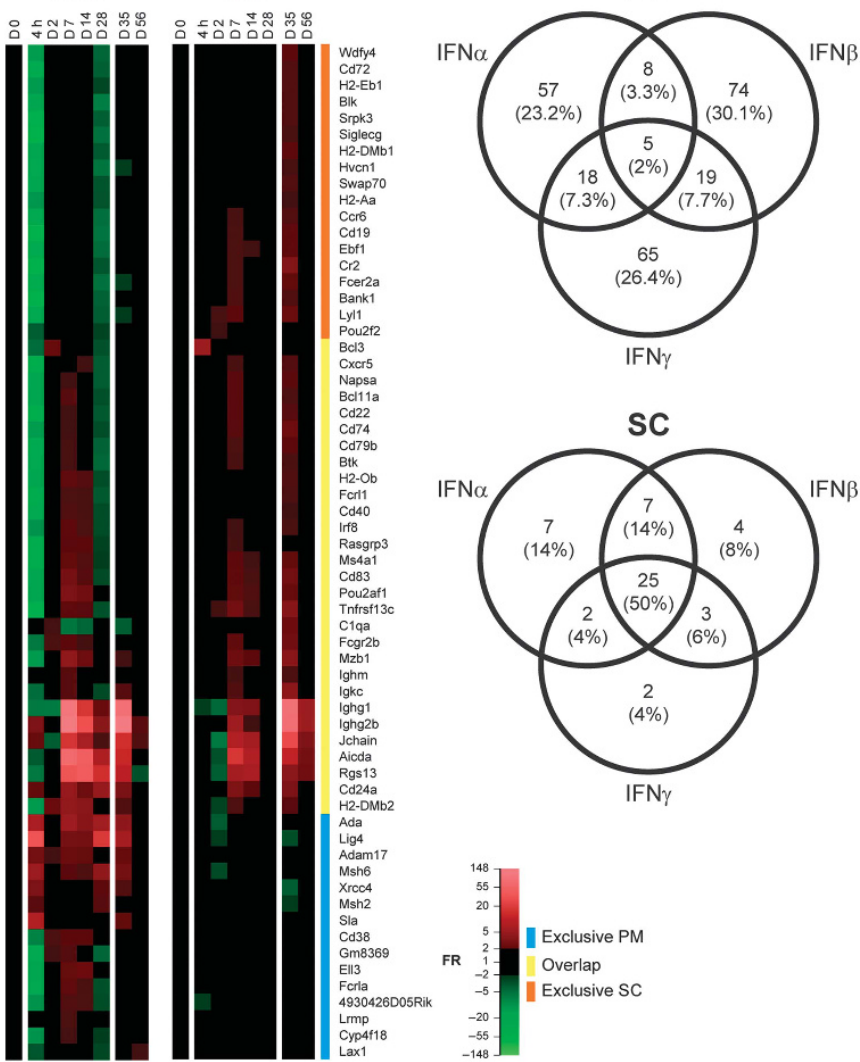

SC
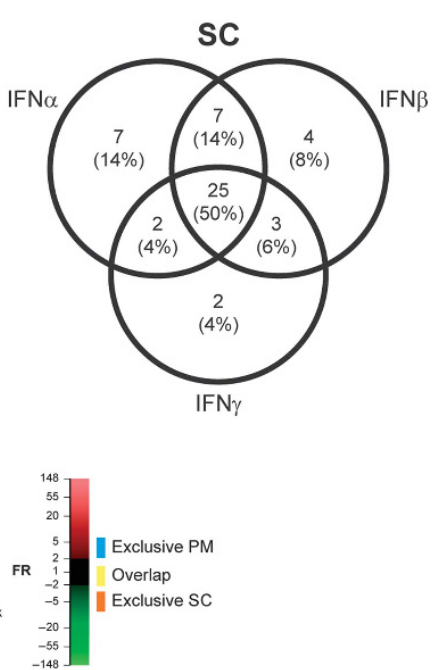

B cell-related genes

Figure 6 Transcriptomic profiles in the draining lymph nodes (dLNs) following pulmonary (PM) and subcutaneous (SC) outer membrane vesicle pertussis vaccine (omvPV) immunization. (a-d) Gene expression in PM and SC mice was compared with naive mice (day 0) (fold ratio (FR) $\geq 1.5, P \leq 0.001$ ). (a) A total of 1,921 genes upregulated (red) or downregulated (green) are visualized in heatmaps (mean of $n=3$ ). Genes not surpassing a fold change of 1.5 are shown as basal level (black). The overlap (yellow) and the exclusive presence of genes in either the PM (blue) or SC (orange) immunization groups is depicted next to the heatmap. (b) Over-representation analysis on all 1,921 genes revealed the involvement of specific Gene Ontology Biological Process (GO-BP) terms with corresponding total amount of genes (blue) and upregulated (red) and downregulated (green) genes. (c) T cell-related genes, including association with distinct Thelper (Th) subsets. (d) B cell-related genes are depicted for PM and SC mice. (e) The numbers and percentages of genes upregulated in the innate phase (4 h) in PM and SC mice are compared with the Interferome database to determine involvement of the type I interferon (IFN; IFN $\alpha$ and IFN $\beta$ ) and/or type II IFN (IFN $\gamma$ ) signaling pathway. 
$\geq 2.0$ ) over time compared with naive mice (Figure 7a). The largest dissimilarity was observed $4 \mathrm{~h}$ after PM immunization, as revealed by principal component analysis (Figure 7b). Many genes were no longer expressed on or after day 2 , although genes were further upregulated (Figure 7a, b). Of note, this early transcriptome profile might be a combination of omvPV-specific and delivery-specific responses that cannot be distinguished in this data set. Pulmonary gene expression on day 28 did not return completely to basal levels. Notably, the lungs of SC mice also showed some differential gene expression, mainly on day 2 (Figure 7a, b). ORA on the DEGs from PM mice revealed enrichment of 127 terms (11 KEGG Pathways and 116 GO-BP terms). A selection of these terms is shown in Figure 7c. Genes involved in pathogen recognition were reflected by expression of pseudo-response regulator (PRR) genes, members of corresponding downstream signaling pathways (inflammasome, TNF, nuclear factor- $\kappa \mathrm{B}$, and mitogen-activated protein kinase), and effector molecules (e.g., those representing proinflammatory responses) (Figure $7 \mathrm{c}$ and Supplementary Figure S7a). Moreover, upregulation was seen in genes expressed in innate lymphoid cells; genes involved in CSF signaling, T-cell activation, and humoral response; and genes encoding complement, cytokines, cytokine receptors, and membrane markers (Figure 7c and Supplementary Figure S7b).

The pulmonary transcriptomic signatures obtained after PM immunization were compared with those obtained after experimental $B$. pertussis infection (558 genes) from a previous study $^{12}$ and revealed 98 overlapping genes. A gene-function network analysis revealed differences in the involvement of genes in all five modules investigated: PRR signaling, acute phase, cytokine response, humoral response, and T-cell responses (Figure 7d). Notably, the infection data set comprised more genes related to acute phase and humoral response, whereas the PM immunization data set included more genes related to cytokine response and PRR signaling. Only 27 shared genes were directly connected to the investigated modules. However, using the STRING database, 42 additional shared genes could be indirectly linked to the modules by their interaction with other genes in the network. A comparison of intensity and kinetics of the average gene expression of each module revealed that PM immunization induced faster and more intense expression of all modules compared with infection (Figure 7e). In conclusion, both PM immunization and $B$. pertussis infection induce similar modules, but with distinct intensity and kinetics of expression and involving different genes.

\section{DISCUSSION}

PM immunization induces local immunity important for protection, as demonstrated for several respiratory pathogens. ${ }^{16-18}$ In this study, immune responses evoked by PM and SC immunization with omvPV were assessed in detail. PM immunization provided superior protection against $B$. pertussis compared with SC immunization, and was associated with distinct innate and adaptive immune signatures. Only PM immunization led to the induction of a mucosal immune response that was indicated by increased gene expression, the presence of particular cytokines, IgA antibodies, IgA- and IgGproducing plasma cells, and tissue-resident Th17 cells in the lungs. Moreover, PM immunization induced a distinct systemic immune response as compared with SC immunization, with fewer IFN-regulated genes in the LNs, lower levels of serum cytokines, and higher serum IgM and IgG levels. PM immunization also increased numbers of $\mathrm{CD} 4^{+} \mathrm{T}$ cells, with a shift in Th subset distribution toward Th17 cells. An overview of the main findings per immunization route is presented in Figure 8 and discussed in detail below.

After PM immunization, expression of genes associated with pathogen recognition was detected in the lungs during the innate phase (Figure 8a). The expression of $L b p, C d 14$, and Tlr2 indicate the involvement of Toll-like receptors 2 and 4 (TLR2 and TLR4), similar to what was observed with whole-cell PV and live bacteria. ${ }^{29-31}$ Interestingly, $T l r 7$ and Nlrp3 were expressed, indicating activation of intracellular PRRs by omvPV. Subsequently, genes downstream of PRRs, including MyD88 and nuclear factor- $\kappa \mathrm{B}$, were expressed. Production of proinflammatory cytokines induced by PRR signaling, such as IL-1 $\beta$, IL-6, and CXCL10, occurred in the lungs on gene and protein levels. Multiple known Th17 effector cytokines ${ }^{13,32}$ and Th17-differentiating cytokines were measured in the lungs, suggesting a Th17-differentiating environment. This was reflected by the presence of Th17 cells in the dLNs, based on expression of Rorc, Hifla, and Havcr1; Th17 cells in the spleen and, more importantly, tissue-resident Th17 cells in the lungs. In addition, Th1-associated cytokines ${ }^{33,34}$ were detected in the lungs and serum, correlating with subsequent detection of systemic Th1 cells. This mixed Th1/Th17 response is considered favorable for providing protection against $B$. pertussis infection. ${ }^{8,12,13}$ In addition, the presence of tissueresident $\mathrm{T}$ cells in the lungs enhances protection against respiratory infections. ${ }^{35}$ The humoral response was characterized by pulmonary expression of IgA-related genes, the IgA transporter (Pigr) and IgA antibodies in lungs and serum. In line with these findings, IgA-producing plasma cells were detected in spleen, blood, and lungs. No IgA-secreting memory B cells were detected in the spleen, most likely because of homing of these cells to other tissues. High serum IgG levels corresponded with specific gene expression (Ighg1, Igh2b, and Jchain) in the dLNs; IgG-producing plasma cells in spleen, blood, and lungs; and IgG-producing memory B cells in the spleen. Interestingly, pulmonary expression of $\mathrm{Nfil3}$ and early expression of Rorc in dLNs suggest involvement of innate lymphoid cells $^{36}$ in the immune response upon PM immunization.

After SC immunization, omvPV is probably recognized in a similar manner as after PM immunization, mainly through TLR2 and TLR4, although this was not investigated at the injection site in this study. The strong systemic cytokine response reveal a mixed Th1-, Th2-, and Th17-differentiating environment that was subsequently reflected by presence of 
a
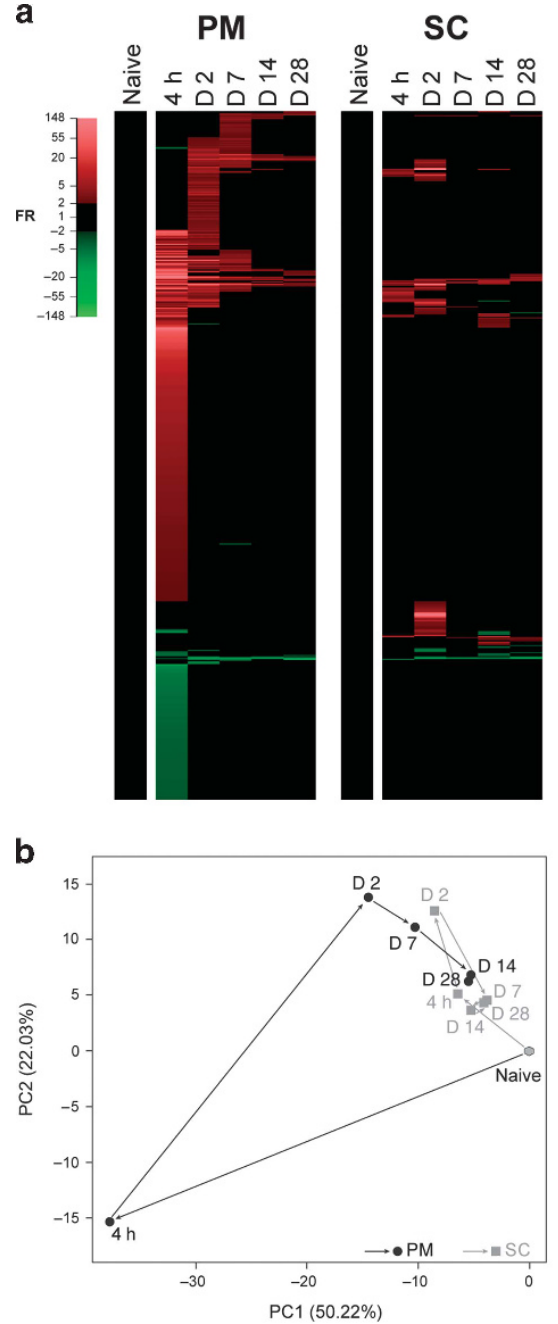

d

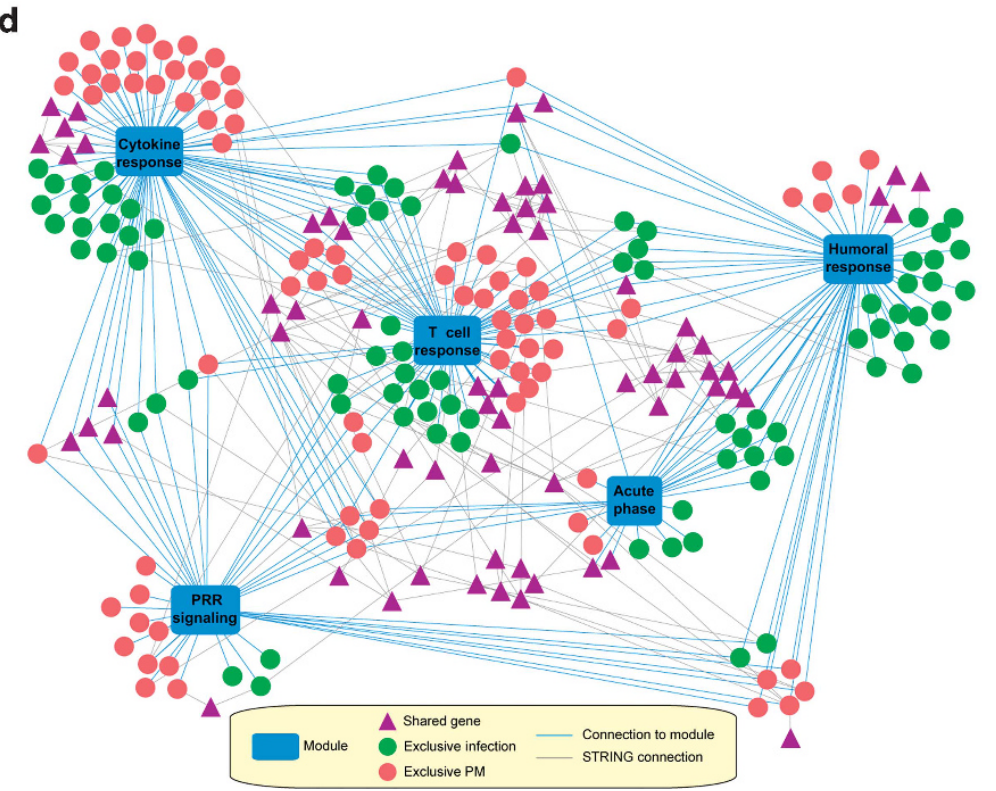

C

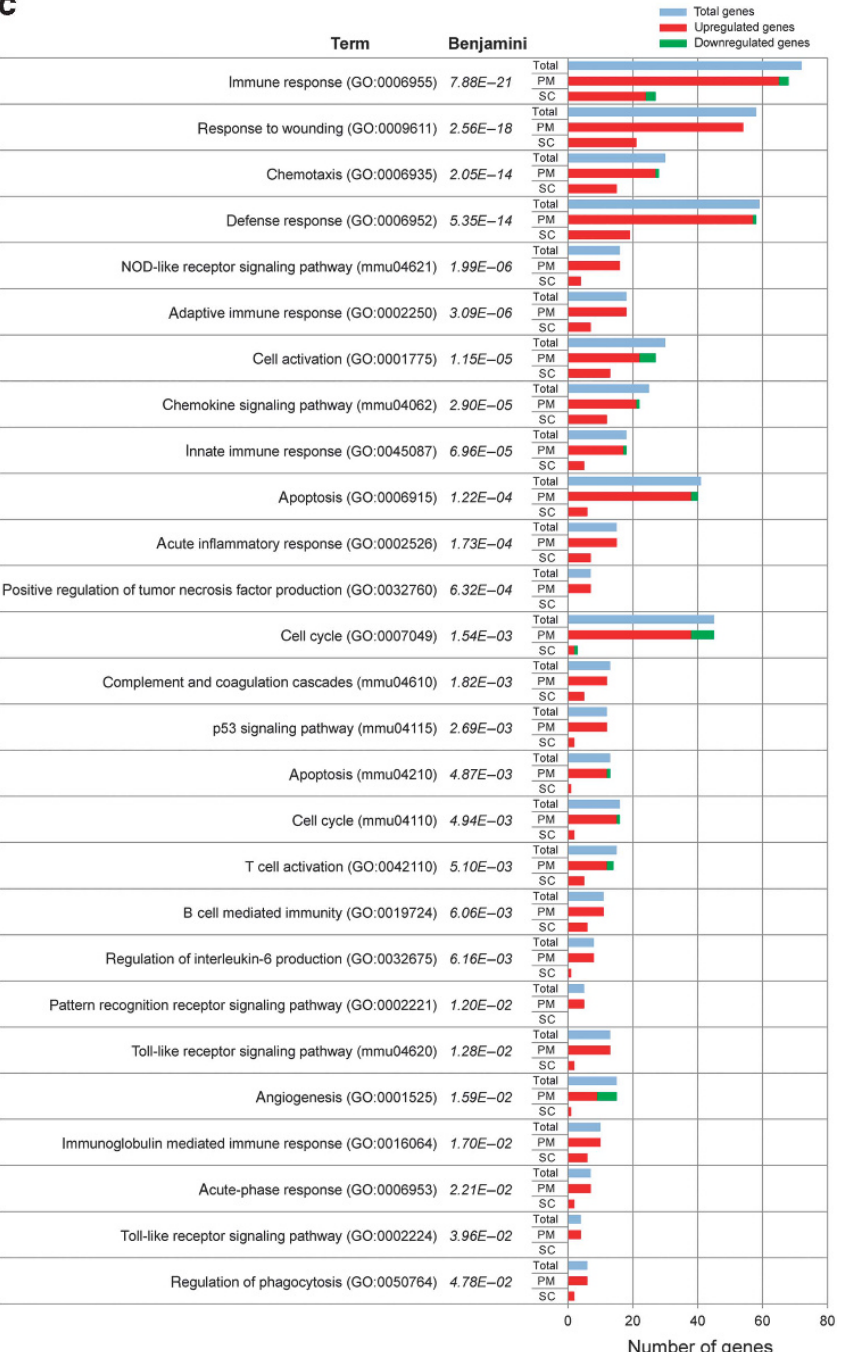


splenic Th1, Th2, and Th17 cells (Figure 8b). Moreover, the induction of Th1 cells was linked to the early expression of genes downstream of IFN signaling in the dLNs. ${ }^{37}$ The source of the early IL-5 production might be type 2 innate lymphoid cells,${ }^{36}$ suggesting their involvement after SC omvPV immunization. Furthermore, expression of B-cell markers (Cd19 and Cd22) and immunoglobulin-related genes (Igh2b, Jchain, and Ighg1) revealed the presence of IgG-producing plasma cells in the dLNs. Accordingly, IgG-producing plasma cells were detected in spleen and blood, IgG-producing memory B cells in spleen, and high IgG levels in serum.

The immunity induced by $\mathrm{PM}$ omvPV immunization substantially resembles infection-induced immunity that remains the benchmark for optimal immunity. Interestingly, $\mathrm{PM}$ omvPV immunization confers protection to a similar extent as an infection in mice. ${ }^{8,12}$ Both PM immunization and

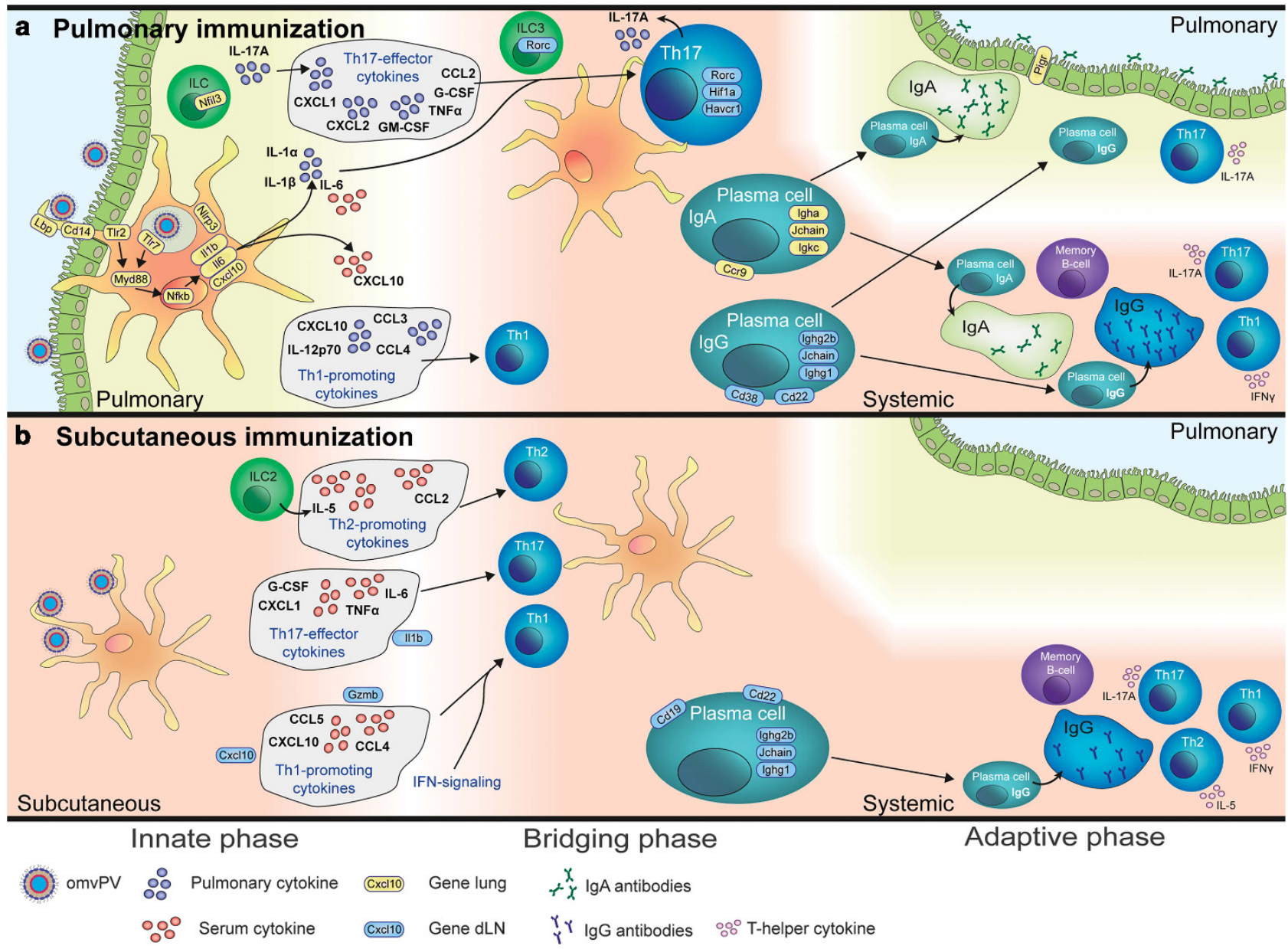

Figure 8 Overview of the immune responses after pulmonary (PM) and subcutaneous (SC) outer membrane vesicle pertussis vaccine (omvPV) immunization. Based on the results obtained in this study, the main findings of the innate and adaptive immune responses were connected for (a) PM immunization and (b) SC immunization.

Figure 7 Pulmonary transcriptomic profiles following pulmonary (PM) and subcutaneous (SC) outer membrane vesicle pertussis vaccine (omvPV) immunization. (a-c) Gene expression was compared with naive mice (day 0 ) (fold ratio (FR) $\geq 2.0, P \leq 0.01$ ). (a) A total of 691 genes upregulated (red) or downregulated (green) are visualized in heatmaps with a mean of $n=3$ for PM mice and $n=1$ (pool of 3 mice) for SC mice. Genes not surpassing a fold ratio of 2.0 are shown as basal level (black). (b) Principal component analysis (PC1 and PC2) shows the (dis)similarity of the five time points for both PM and SC mice. (c) Over-representation analysis (ORA) on 691 genes revealed involvement of specific Gene Ontology Biological Process (GO-BP) terms and KEGG (Kyoto Encyclopedia of Genes and Genomes) pathways with corresponding total amount (blue), upregulated (red), and downregulated (green) genes. (d, e) Pulmonary transcriptomes were compared after PM immunization and Bordetella pertussis infection. ${ }^{12}$ (d) A gene-function network analysis showing the connection of genes expressed within 28 days after PM immunization/infection to a specific module (blue line) based on involvement in acute phase, cytokine response, humoral response, pathogen recognition receptor (PRR) signaling, and T-cell responses. Genes expressed in both data sets (shared, triangle, purple), exclusively expressed in infected mice (circle, green), or exclusively expressed in PM mice (circle, red) are depicted. In addition, indirect linkage of shared genes with other genes in the network (gray line) by using the STRING database are shown. (e) For infected mice (9 time points) and PM mice (6 time points), an average fold ratio (expressed as LN-transformed numbers) was calculated to determine the expression intensity and kinetics for each module corresponding to those included in the network analysis. 
B. pertussis infection induce a pulmonary $\operatorname{IgA}$ response. Moreover, the pulmonary and systemic $\mathrm{CD} 4{ }^{+} \mathrm{T}$-cell responses are similar and Th17 dominated. ${ }^{8,12,13}$

Still, considerable differences were found between PM immunization and infection-induced immunity. Serum IgG and pulmonary IgA levels seem higher after the booster omvPV PM immunization compared with the levels observed 56 days after infection; ${ }^{10,14}$ however, direct side-by-side studies are required to confirm this. Compared with infection, the omvPV immunization in general evoked a distinct IgG subclass response caused in part by high anti-lipopolysaccharide IgG3 induction after the booster immunization. IgG subclass distribution is considered indirect evidence for Th responses. ${ }^{38-40}$ In our study, the Th responses are not mirrored in the IgG subclass responses, as the Th responses are comparable after PM immunization and infection, whereas the IgG subclass distribution is distinct. As both SC and PM administration of omvPV result in a comparable IgG subclass response, the differences compared with infection probably reflect vaccine composition and vaccination scheme, not the administration route. Furthermore, the current and previous study ${ }^{10}$ together demonstrate that distinct antigen composition likely explains the difference between antigen specificity of serum IgG induced by PM immunization vs. infection. Whereas omvPV contains mainly outer membrane and periplasmic proteins, live bacteria comprise additional cytosolic proteins and are capable of de novo production and secretion of antigens, such as pertussis toxin (Ptx) ${ }^{10}$ or antigens expressed only when infecting a host and not during cultivation for vaccine production.

PM immunization leads to faster and more intense pulmonary and serum cytokine production as compared with infection. In fact, higher cytokine levels could have been expected during $B$. pertussis infection because of a prolonged antigen exposure ${ }^{12}$ and higher antigen dose compared with PM immunization with non-replicating omvPV. Therefore, weaker cytokine responses upon an infection most likely could be explained by the immune evasion strategies of B. pertussis, such as the dampening of host cytokine responses. ${ }^{41}$ Extensive comparison of early pulmonary transcriptomic profiles induced by PM immunization obtained from this study vs. those induced by $B$. pertussis infection ${ }^{12}$ demonstrated that although both responses included genes involved in the acute phase, PRR signaling, cytokine responses, humoral response, and T-cell responses, these responses involved distinct genes. Moreover, PM immunization led to faster and more intense pulmonary gene expression, in line with cytokine profiles. Importantly, in contrast to $\mathrm{PM}$ omvPV immunization, $B$. pertussis colonization in the respiratory tract during infection is a dynamic process that may lead to a distinct immune stimulation and may explain some of the observed differences.

In general, the comparison of omvPV-induced immunity through PM administration vs. infection-induced immunity revealed overall comparability, notwithstanding some distinct innate responses.
An advantage of pulmonary immunization, besides the induction of mucosal immune responses resembling that induced by $B$. pertussis infection, is that the administration is needle-free. Currently, no pulmonary vaccines and very few mucosal vaccines have been approved for human use. The latter include a nasal vaccine against influenza virus and oral vaccines against poliovirus, rotavirus, Salmonella typhi, and Vibrio cholerae. ${ }^{42}$ Pulmonary vaccine administration in humans is in early clinical development. Both powder inhalers ${ }^{43}$ and fluid and nebulized vaccines have been clinically tested, the latter in infants as young as 9 months of age. ${ }^{44}$ Intranasal vaccine administration might also be an option as intranasal omvPV immunization also provides protection against $B$. pertussis infection ${ }^{19}$ and may be better suitable for infants. Intranasal administration of a meningococcal OMV vaccine in humans demonstrated induction of mucosal antibodies. ${ }^{45}$ In addition, intranasal administration results not only in IgA antibodies in the nasal cavity but also in the lower respiratory tract. ${ }^{42,46} \mathrm{In}$ depth analysis of immune responses induced by intranasal immunization has still to be performed with pertussis vaccines.

This study confirms the potential of omvPV as a novel pertussis vaccine candidate. ${ }^{10,11,19}$ A different administration route further enhanced the vaccine performance. In addition, it is possible to include or alter more antigens, besides pertactin, to further improve the vaccine. For example, if the presence of Ptx is critical for the induction of efficient protection, this could be added. A broad antigenic composition in omvPV ${ }^{10}$ may also limit the emergence of strains lacking antigens because of vaccination-induced selection, as has occurred for pertactin, likely in response to introduction of acellular pertussis vaccine.

In conclusion, this study showed that PM immunization of omvPV elicits superior local and systemic immunity against $B$. pertussis infection compared with SC immunization. Moreover, the adaptive immune response induced by $\mathrm{PM}$ omvPV immunization is to a large extent comparable to that induced by a $B$. pertussis infection. Therefore, mucosal immunization may be key to improved protection against $B$. pertussis infection and accordingly must be considered in the development of novel candidate pertussis vaccines. In addition to serology, which is still the gold standard in pertussis research, a systems vaccinology approach is invaluable in order to unravel and understand the immunological signatures underlying vaccineinduced immunity. Such understanding facilitates a rational choice of a vaccine candidate and route of administration, mitigating the risk of vaccine failure in the late stage of development.

\section{METHODS}

Vaccine and antigens. The omvPV was prepared ${ }^{11}$ using a genetically modified B. pertussis B1917 strain with retained Prn. ${ }^{27}$ B. pertussis antigens Ptx and filamentous hemagglutinin (FHA) were obtained from Kaketsuken (Kumamoto, Japan). Prn and fimbriae (Fim) 2/3 were kindly provided by Betsy Kuipers (RIVM, Bilthoven, The Netherlands). 
Immunization and challenge of mice. An independent ethical committee for animal experimentation of Intravacc approved the animal experiments. Animal handling in this study was carried out in accordance with the guidelines provided by the Dutch Act on Animal Experimentation. Mice within groups were randomly divided after receiving the vaccine via a particular administration route or the $B$. pertussis infection to prevent subjective bias. On days 0 and 28, 8-weekold female $\mathrm{BALB} / \mathrm{c}$ mice (Harlan, Horst, The Netherlands) were immunized with omvPV containing $1 \mu \mathrm{g}$ total protein in $50 \mu \mathrm{l}$ phosphate-buffered saline (PBS) for the PM route or $300 \mu \mathrm{l}$ PBS for the SC route. NI mice were used as a control. Pulmonary administration was performed as described by Bivas-Benita et al. ${ }^{47}$ using a MicroSprayer aerosolizer supplied with a high-pressure syringe (IA-1C and FMJ-250; Penn-Century, Philadelphia, PA). On day 56, mice were challenged intranasally under anesthesia (isoflurane/oxygen) with $2 \times 10$ E5 colony-forming units of B. pertussis B1917 in $20 \mu$ of THIJS medium. The $B$. pertussis challenge culture was prepared as described in the literature, ${ }^{12}$ except that bacteria were grown in THIJS medium. ${ }^{48}$

A study design overview with the number of animals, tissue collection, and performed methods for each group and time point is schematically depicted in Figure 1a.

Sample collection. Mice were anesthetized (isoflurane/oxygen) for orbital blood collection and killed by cervical dislocation. From some mice, the left lung lobes were incubated in RNAlater (Qiagen, Hilden, Germany) and stored at $-80^{\circ} \mathrm{C}$ for microarray analysis. The right lung lobes and trachea, used for antibody detection and colonization assays, were homogenized in THIJS medium using a Bio-Gen PRO200 Homogenizer (Pro Scientific, Oxford, CT) and nose lavages were obtained (after removal of the trachea) by flushing the nose with THIJS medium.

Complete lungs of other mice and spleens for T- and B-cell assays, and dLNs (bronchial LNs for PM and inguinal LNs for SC immunization) for microarray analysis were collected in RPMI complete medium (RPMI-1640 medium (Thermo Fisher, Waltham, MA) supplemented with $10 \%$ fetal calf serum (GE Healthcare, Hoevelaken, The Netherlands), 100 units penicillin, 100 units streptomycin, and $2.92 \mathrm{mg} \mathrm{ml}^{-1}$ L-glutamine (Thermo Fisher)) and homogenized using a $70 \mu \mathrm{m}$ cell strainer (BD Falcon, Vianen, The Netherlands). Erythrocytes in spleen and lung samples were lysed using homemade lysis buffer $\left(10 \mathrm{gl}^{-1} \mathrm{NH}_{4} \mathrm{CL}, 1.25 \mathrm{gl}^{-1} \mathrm{NaHCO}_{3}\right.$, $0.125 \mathrm{mM}$ EDTA in $\mathrm{dH}_{2} 0, \mathrm{pH} 7.4$ ).

Whole blood was collected in heparinized tubes (MiniCollect $1 \mathrm{ml}$ LH, Greiner, Kremsmünster, Austria) after which erythrocytes were lysed using RBC lysis buffer (Pharm Lyse, BD; B-cell ELISpot (enzymelinked immunospot)) or the homemade lysis buffer (tetramer assay).

Serum was obtained by collecting whole blood in a serum collection tube (MiniCollect $0.8 \mathrm{ml} \mathrm{Z} \mathrm{Serum} \mathrm{Sep} \mathrm{GOLD,} \mathrm{Greiner).} \mathrm{After}$ coagulation (10 $\mathrm{min}$, room temperature) and centrifugation $(10 \mathrm{~min}, 3,000 \mathrm{~g})$, sera were collected, aliquoted in single vials for each analysis, and stored at $-80^{\circ} \mathrm{C}$.

Colonization assays. Lung and trachea lysates, and nose lavages were serially diluted (undiluted, 1:10, 1:100, and 1:1,000) in THIJS medium, plated on Bordet-Gengou agar plates and incubated $\left(5\right.$ days, $\left.35^{\circ} \mathrm{C}\right)$. The colony-forming units per $\mathrm{ml}$ were determined using a colony counter (ProtoCOL, Synbiosis, Cambridge, UK).

B-cell ELISpot. Filter plates (Multiscreen-HA 96-well plates, Millipore, Amsterdam, The Netherlands) were coated (overnight, $4{ }^{\circ} \mathrm{C}$ ) with $5 \mu \mathrm{g} \mathrm{ml}^{-1}$ Prn or $10 \mu \mathrm{g} \mathrm{ml}^{-1}$ wild-type B1917 OMV. Prn and B1917 OMVs (containing only low amounts of Prn) ${ }^{10}$ were used for stimulation to optimally distinguish B-cell responses directed to Prn from responses to other antigens in omvPV. Wells were coated with a goat-anti-mouse kappa- and lambda-mixture $\left(7 \mu \mathrm{g} \mathrm{ml}^{-1}\right.$ each) (Southern Biotech, Birmingham, AL) as positive control, or left uncoated (PBS) as negative control. After washing with PBS, plates were blocked with RPMI-1640 + 2\% Protifar (Nutricia,
Zoetermeer, The Netherlands) (1 h, room temperature) and washed again.

For memory B-cell analysis, splenocytes were stimulated $\left(5 \times 10^{5}\right.$ cells per well, 24 -well plate; 5 days, $37^{\circ} \mathrm{C}$ ) with $10 \mu \mathrm{g} \mathrm{ml}^{-1} \mathrm{CpG}$ ODN 1826 (Invivogen, San Diego, CA), $10 \mu \mathrm{g} \mathrm{ml}^{-1}$ pokeweed mitogen (Sigma-Alderich, Zwijndrecht, The Netherlands), and Staphylococcus aureus protein A of Cowan Strain (1:5,000; Sigma) in RPMI complete medium with $\beta$-mercaptoethanol (1:25,000; Sigma) to induce antibody secretion. ${ }^{49}$ Cells from blood $\left(0.75 \times 10^{5}\right.$ cells per well), lungs $\left(0.75 \times 10^{5}\right.$ cells per well $)$, spleen $\left(5 \times 10^{5}\right.$ cells per well $)$, or stimulated splenocytes $\left(5 \times 10^{5}\right.$ cells per well $)$ were added to coated plates and incubated (overnight, $37^{\circ} \mathrm{C}$ ). Plates were washed with PBS and PBS-T $\left(0.05 \%\right.$ Tween-20) and then incubated $\left(1 \mathrm{~h}, 37^{\circ} \mathrm{C}\right)$ with alkaline phosphatase-conjugated goat-anti-mouse IgA or IgG (Southern Biotech; 1:1,000). Plates were washed with PBS, PBS-T, and tap water. Subsequently, spot development was initiated with $0.45 \mu \mathrm{m}$ filtered BCIP-NBT substrate (Sigma) and stopped by extensive rinsing with distilled water. Spots were counted with an AID iSpot reader (Autoimmun Diagnostika, Strassberg, Germany) and indicated as antibody-secreting cells per $5 \times 10^{5}$ cells.

Antibody measurements. Antibodies specific for OMV, Prn, FHA, Ptx, and Fim2/3 were measured using a previously described mouse multiplex immunoassay. ${ }^{10}$ Serum samples were diluted 1:5,000 for IgG (subclass) and 1:100 for IgM and IgA measurements. Lung lysates were diluted 1:100 for measuring IgA levels. Data were acquired with a Bio-Plex 200, processed using Bio-Plex Manager software (v5.0, Bio-Rad Laboratories, Veenendaal, The Netherlands), and presented as fluorescence intensities.

Detection of Prn $_{10-24}$-specific CD4 ${ }^{+}$T cells. Cells from erythrocytelysed blood were stained $\left(1 \mathrm{~h}, 37^{\circ} \mathrm{C}\right)$ with $\operatorname{Prn}_{10-24}$-specific APCconjugated I-A ${ }^{\mathrm{d}}$ tetramers $^{50}$ (NIH Tetramer Facility, Atlanta, GA) in RPMI complete medium. Next, cells were stained $\left(30 \mathrm{~min}, 4^{\circ} \mathrm{C}\right)$ with Pacific blue-conjugated anti-CD4 (Biolegend, London, UK), FITCconjugated anti-CD44 (BD), and LIVE/DEAD Fixable Aqua Dead Cell Stain Kit in FACS buffer (PBS; pH 7.2, 0.5\% bovine serum albumin (Sigma Aldrich), 0.5 mM EDTA (ICN Biomedicals, ICN Biomedicals, Santa Ana, CA)). Data were acquired on a FACS Canto II (BD) and analyzed using FlowJo software (Tree Star, Ashland, OR).

Intracellular cytokine staining. Cells from lungs and spleen were stimulated $\left(6 \times 10^{6}\right.$ cells per well, 24 -well plates, 3 days, $\left.37^{\circ} \mathrm{C}\right)$ with $1 \mu \mathrm{g} \mathrm{ml}{ }^{-1}$ Prn or $1.5 \mu \mathrm{g} \mathrm{ml}^{-1}$ wild-type B1917 OMVs in Iscove's modified Dulbecco's medium (IMDM) complete medium (IMDM (Gibco), 8\% fetal calf serum, 100 units penicillin, 100 units streptomycin, $2.92 \mathrm{mg} \mathrm{ml}^{-1} \quad$ L-glutamine, $20 \mu \mathrm{M} \quad \beta$-mercaptoethanol (Sigma)). Prn and B1917 OMVs (containing only low amounts of Prn $)^{10}$ were used for stimulation to optimally distinguish T-cell responses directed to Prn from responses to other antigens in omvPV. On day 3, supernatant was collected for cytokine analysis; cells were transferred to U-bottom 96 -well plates $\left(5 \times 10^{5}\right.$ cells per well $)$ and restimulated overnight using the same antigen conditions.

ICS was performed using the BD Cytofix/Cytoperm Fixation/ Permeabilization Solution Kit (BD). Cells were incubated with $10 \mu \mathrm{g} \mathrm{ml}^{-1}$ Golgiplug (BD), $1 \mu \mathrm{g} \mathrm{ml}^{-1}$ anti-CD28 (BD), and $1 \mu \mathrm{g} \mathrm{ml}^{-1}$ anti-CD49d (BD) during the last $5 \mathrm{~h}$ of restimulation. Cells were then stained with Pacific blue-conjugated anti-CD4 (Biolegend), FITC-conjugated anti-CD44 (BD), PE-Cy7-conjugated anti-CD103 (Biolegend; only lung cells), and LIVE/DEAD Fixable Aqua Dead Cell Stain (Invitrogen). Thereafter, cells were fixed, permeabilized, and stained with PE-conjugated anti-IFN $\gamma$ (BD), APCconjugated anti-IL-5 (Biolegend), and PerCP-Cy5.5-conjugated antiIL-17A (eBioscience). Data were acquired on a FACS Canto II and processed using FlowJo software.

Multiplex cytokine analysis. In serum and lung lysates, concentrations of 32 cytokines were determined using a Milliplex mouse 
Cytokine/Chemokine 32-plex luminex kit (Millipore). The concentration of various Th subset cytokines (IL-4, IL-5, IL-10, IL-13, IL$17 \mathrm{~A}, \mathrm{TNF} \alpha$, and IFN $\gamma$ ) was determined in splenic culture supernatant using a Milliplex mouse cytokine luminex kit (Millipore). Measurements and data analysis were performed with a Bio-Plex 200 and using Bio-PlexManager software (v5.0, Bio-Rad). Results were corrected for the background (IMDM complete medium control) per mouse per stimulation per cytokine, and calculated in $\mathrm{pg} \mathrm{ml}^{-1}$.

RNA isolation and microarray analysis. RNA isolation from lung tissue with additional determination of RNA concentrations and integrity was performed as described previously. ${ }^{12}$ RNA from cells in the dLNs was isolated using a MagNA Pure LC RNA Isolation High Performance kit and MagNA Pure System (Roche, Basel, Switzerland). For lung tissue, RNA concentrates from naive and PM mice were analyzed as individual samples, whereas those of SC mice were pooled.

Amplification, labeling, and hybridization of RNA for microarray (HT MG-430 PM Array Plates, Affymetrix, Santa Clara, CA) were performed at the Microarray Department of the University of Amsterdam, The Netherlands. Complete raw and normalized microarray data and their MIAME compliant metadata were submitted to the Gene Expression Omnibus (GEO) database (www.ncbi.nlm.nih.gov/geo) with identifiers GSE83602 and GSE83603.

Transcriptomic data analysis. Quality control and normalization of raw Affymetrix CEL files were performed at ArrayAnalysis (www.arrayanalysis.org) ${ }^{51}$ using the Robust Multichip Average method $^{52}$ and the MBNI custom CDF (v.19). ${ }^{53}$ Analysis of normalized data, consisting of Log2-transformed signal values for 17,856 genes, was performed in R (www.r-project.org) and Microsoft Excel. Analysis of variance was applied to identify DEGs between experimental groups before immunization and various time points afterward. The induction or repression of individual genes was expressed as a FR by comparing mean gene expression levels in experimental groups vs. naive mice. The DEG criteria for transcriptome analysis on lung tissue were $p<0.01$ (analysis of variance), FR $>2.0$ and for $\operatorname{dLN} p<0.001$, FR $>1.5$. GeneMaths XT (Applied Maths, St-Martens-Latem, Belgium) was used to visualize the heatmaps. Genes were arranged according to similar expression patterns over time at which genes exceeded FR cutoffs. Principal component analysis based on expression profiles of all DEGs was performed in R. Functional enrichment based on GO-BP and KEGG was performed using ORA in DAVID. Involvement of type I and II IFN signaling pathways was analyzed using the Interferome database (http://www.interferome.org/ interferome/home.jspx). ${ }^{54}$

Gene network analysis. To construct a gene-function network, ORA results and additional text mining were used to determine genes associated with five modules: acute phase, cytokine response, humoral response, PRR signaling, and T-cell responses. Interactions between genes were determined using STRING (http://string.embl.de/) with high confidence (0.700) and using co-occurrence, coexpression, experiments, databases, and text mining as types of evidence. Genefunction associations and gene-gene interactions were combined into one network file. The network visualization was performed using Cytoscape (v.2.8.3; http://www.cytoscape.org/).

Immunoproteomic profiling. To identify antigen specificity of antibody responses, electrophoresis combined with western blotting and liquid chromatography-mass spectrometry analysis were performed. ${ }^{10}$ The B. pertussis for lysate and omvPV were cultivated and harvested under similar conditions (defined medium, optical density, similar strain) to ensure overlap of all antigens. Gels were blotted with respectively $10-15 \mu \mathrm{g}$ and $25 \mu \mathrm{g}$ protein of a B. pertussis (B1917) lysate for one- and two-dimensional electrophoresis. Blots were treated with diluted sera $(1: 100)$ or diluted lung lysates (1:10-1:50) before immunostaining with IR-800-labeled goat-anti-mouse IgG or IgA. Blots were scanned using an Odyssey infrared imager (Westburg,
Leusden, The Netherlands) and analyzed with Delta2D (v.4.5, Decodon, Greifswald, Germany).

Statistics. To determine significance of intergroup differences for B-cell ELISpot, T-cell ICS, and T-cell tetramer analysis, MannWhitney $t$-test was used. Data from cytokine, antibody, and colonization assays were log-transformed and statistically tested ( $t$-test). $P$ values of $\leq 0.05$ were considered as significant differences.

SUPPLEMENTARY MATERIAL is linked to the online version of the paper at http://www.nature.com/mi

\section{ACKNOWLEDGMENTS}

We are thankful to Tim Bindels and employees of the Animal Research Centre (ARC) (Intravacc, Bilthoven, The Netherlands) for respectively the production of the omvPV and performance of animal experiments. We are grateful to Dale Long and Rick Willis (NIH Tetramer Facility, Atlanta, USA) for supplying the tetramers. Finally, we thank Lucy Phillips for providing editorial review of the manuscript.

\section{AUTHOR CONTRIBUTIONS}

R.H.M.R., J.B., C.A.C.M.E., G.F.A.K., W.G.H.H., and B.M. conceived the experiments; R.H.M.R., J.B., L.M., W.T., and K.H. conducted the experiments; R.H.M.R., J.B., J.L.A.P., L.M., W.T., and K.H. analyzed the results; R.H.M.R., J.B, E.R., C.A.C.M.E., G.F.A.K. W.G.H.H., and B.M. wrote the main manuscript; J.L.A.P., L.M., W.T., K.H., A.A., A.S., P.L., W.E., and W.J. reviewed the manuscript.

\section{DISCLOSURE}

The authors declared no conflict of interest.

Official journal of the Society for Mucosal Immunology

\section{REFERENCES}

1. Park, C.O. \& Kupper, T.S. The emerging role of resident memory T cells in protective immunity and inflammatory disease. Nat. Med. 21, 688-697 (2015).

2. Mueller, S.N. \& Mackay, L.K. Tissue-resident memory T cells: local specialists in immune defence. Nat. Rev. Immunol. 16, 79-89 (2016).

3. Chiu, C. \& Openshaw, P.J. Antiviral B cell and T cell immunity in the lungs. Nat. Immunol. 16, 18-26 (2015).

4. Rumbo, M. \& Hozbor, D. Development of improved pertussis vaccine. Hum. Vaccin. Immunother. 10, 2450-2453 (2014).

5. Brummelman, J., Wilk, M.M., Han, W.G., van Els, C.A. \& Mills, K.H. Roads to the development of improved pertussis vaccines paved by immunology. Pathog. Dis. 73, ftv067 (2015).

6. Mascart, F., Hainaut, M., Peltier, A., Verscheure, V., Levy, J. \& Locht, C. Modulation of the infant immune responses by the first pertussis vaccine administrations. Vaccine 25, 391-398 (2007).

7. Vermeulen, F. et al. Cellular immune responses of preterm infants after vaccination with whole-cell or acellular pertussis vaccines. Clin. Vaccine Immunol. 17, 258-262 (2010).

8. Ross, P.J. et al. Relative contribution of Th1 and Th17 cells in adaptive immunity to Bordetella pertussis: towards the rational design of an improved acellular pertussis vaccine. PLoS Pathog. 9, e1003264 (2013).

9. Brummelman, J. et al. Modulation of the CD4 $(+)$ T cell response after acellular pertussis vaccination in the presence of TLR4 ligation. Vaccine 33, 1483-1491 (2015).

10. Raeven, R.H.M. et al. Immunoproteomic profiling of Bordetella pertussis outer membrane vesicle vaccine reveals broad and balanced humoral immunogenicity. J. Proteome Res. 14, 2929-2942 (2015).

11. Raeven, R.H.M. et al. Bordetella pertussis outer membrane vesicle vaccine confers equal efficacy in mice with milder inflammatory responses compared to a whole-cell vaccine. Sci. Rep. 6, 38240 (2016).

12. Raeven, R.H.M. et al. Molecular signatures of the evolving immune response in mice following a Bordetella pertussis infection. PLOS ONE 9, e104548 (2014) 
13. Warfel, J.M. \& Merkel, T.J. Bordetella pertussis infection induces a mucosal $\mathrm{IL}-17$ response and long-lived Th17 and Th1 immune memory cells in nonhuman primates. Mucosal Immunol. 6, 787-796 (2013).

14. Raeven, R.H.M. et al. Immunological signatures after Bordetella pertussis infection demonstrate importance of pulmonary innate immune cells. PLoS ONE 11, e0164027 (2016).

15. Ogra, P.L. \& Karzon, D.T. Poliovirus antibody response in serum and nasal secretions following intranasal inoculation with inactivated poliovaccine. J. Immunol. 102, 15-23 (1969).

16. Aguilo, N. et al. Pulmonary but not subcutaneous vaccination confers protection to TB susceptible mice by an IL17-dependent mechanism. J. Infect. Dis. 213, 831-839 (2015).

17. Liu, H., Patil, H.P., de Vries-Idema, J., Wilschut, J. \& Huckriede, A. Evaluation of mucosal and systemic immune responses elicited by GPI0100- adjuvanted influenza vaccine delivered by different immunization strategies. PLOS ONE 8, e69649 (2013).

18. Wang, J. et al. Single mucosal, but not parenteral, immunization with recombinant adenoviral-based vaccine provides potent protection from pulmonary tuberculosis. J. Immunol. 173, 6357-6365 (2004).

19. Roberts, R. et al. Outer membrane vesicles as acellular vaccine against pertussis. Vaccine 26, 4639-4646 (2008)

20. Asensio, C.J. et al. Outer membrane vesicles obtained from Bordetella pertussis Tohama expressing the lipid A deacylase PagL as a novel acellular vaccine candidate. Vaccine 29, 1649-1656 (2011).

21. Asokanathan, C., Corbel, M. \& Xing, D. A CpG-containing oligodeoxynucleotide adjuvant for acellular pertussis vaccine improves the protective response against Bordetella pertussis. Hum. Vaccin. Immunother. 9, 325-331 (2013).

22. Mielcarek, N. et al. Live attenuated B. pertussis as a single-dose nasal vaccine against whooping cough. PLoS Pathog. 2, e65 (2006).

23. Querec, T.D. et al. Systems biology approach predicts immunogenicity of the yellow fever vaccine in humans. Nat. Immunol. 10, 116-125 (2009).

24. Li, S. et al. Molecular signatures of antibody responses derived from a systems biology study of five human vaccines. Nat. Immunol. 15, 195-204 (2014).

25. Furman, D. et al. Apoptosis and other immune biomarkers predict influenza vaccine responsiveness. Mol. Syst. Biol. 9, 659 (2013).

26. Nakaya, H.I. et al. Systems biology of vaccination for seasonal influenza in humans. Nat. Immunol. 12, 786-795 (2011).

27. Dautin, N., Barnard, T.J., Anderson, D.E. \& Bernstein, H.D. Cleavage of a bacterial autotransporter by an evolutionarily convergent autocatalytic mechanism. EMBO J. 26, 1942-1952 (2007).

28. Bart, M.J., Zeddeman, A., van der Heide, H.G., Heuvelman, K., van Gent, M. \& Mooi, F.R. Complete genome sequences of Bordetella pertussis isolates B1917 and B1920, representing two predominant global lineages. Genome Announc. 2, pii: e01301-14 (2014).

29. Brummelman, J. et al. Bordetella pertussis naturally occurring isolates with altered lipooligosaccharide structure fail to fully mature human dendritic cells. Infect. Immun. 83, 227-238 (2015).

30. Banus, S. et al. The role of Toll-like receptor-4 in pertussis vaccine-induced immunity. BMC Immunol. 9, 21 (2008).

31. Higgins, S.C., Jarnicki, A.G., Lavelle, E.C. \& Mills, K.H. TLR4 mediates vaccine-induced protective cellular immunity to Bordetella pertussis: role of IL-17-producing T cells. J. Immunol. 177, 7980-7989 (2006).

32. Tsai, H.C., Velichko, S., Hung, L.Y. \& Wu, R. IL-17A and Th17 cells in lung inflammation: an update on the role of Th17 cell differentiation and IL-17R signaling in host defense against infection. Clin. Dev. Immunol. 2013, 267971 (2013).

33. Rot, A. \& von Andrian, U.H. Chemokines in innate and adaptive host defense: basic chemokinese grammar for immune cells. Annu. Rev. Immunol. 22, 891-928 (2004).

34. Christie, D. \& Zhu, J. Transcriptional regulatory networks for CD4 T cell differentiation. Curr. Top. Microbiol. Immunol. 381, 125-172 (2014).

35. Turner, D.L. \& Farber, D.L. Mucosal resident memory CD4 T cells in protection and immunopathology. Front. Immunol. 5, 331 (2014).

36. Zhong, C. \& Zhu, J. Transcriptional regulatory network for the development of innate lymphoid cells. Mediators Inflamm. 2015, 264502 (2015).
37. Huber, J.P. \& Farrar, J.D. Regulation of effector and memory T-cell functions by type I interferon. Immunology 132, 466-474 (2011).

38. Mitsdoerffer, M. et al. Proinflammatory $T$ helper type 17 cells are effective B-cell helpers. Proc. Natl. Acad. Sci. USA 107, 14292-14297 (2010).

39. Stevens, T.L. et al. Regulation of antibody isotype secretion by subsets of antigen-specific helper T cells. Nature 334, 255-258 (1988).

40. Germann, T. et al. Interleukin-12 profoundly up-regulates the synthesis of antigen-specific complement-fixing lgG2a, IgG2b and IgG3 antibody subclasses in vivo. Eur. J. Immunol. 25, 823-829 (1995).

41. Andreasen, C. \& Carbonetti, N.H. Pertussis toxin inhibits early chemokine production to delay neutrophil recruitment in response to Bordetella pertussis respiratory tract infection in mice. Infect. Immun. 76, 5139-5148 (2008).

42. Holmgren, J. \& Czerkinsky, C. Mucosal immunity and vaccines. Nat. Med. 11 (4 Suppl), S45-S53 (2005).

43. Cape, S. et al. Safety and immunogenicity of dry powder measles vaccine administered by inhalation: a randomized controlled Phase I clinical trial. Vaccine 32, 6791-6797 (2014).

44. Wong-Chew, R.M. et al. Immunogenicity of aerosol measles vaccine given as the primary measles immunization to nine-month-old Mexican children. Vaccine 24, 683-690 (2006).

45. Haneberg, B. et al. Intranasal administration of a meningococcal outer membrane vesicle vaccine induces persistent local mucosal antibodies and serum antibodies with strong bactericidal activity in humans. Infect. Immun. 66, 1334-1341 (1998).

46. Bergquist, C., Lagergard, T., Lindblad, M. \& Holmgren, J. Local and systemic antibody responses to dextran-cholera toxin B subunit conjugates. Infect. Immun. 63, 2021-2025 (1995).

47. Bivas-Benita, M., Zwier, R., Junginger, H.E. \& Borchard, G. Non-invasive pulmonary aerosol delivery in mice by the endotracheal route. Eur. J. Pharm. Biopharm. 61, 214-218 (2005).

48. Thalen, M. et al. Rational medium design for Bordetella pertussis: basic metabolism. J. Biotechnol. 75, 147-159 (1999).

49. Crotty, S. \& Ahmed, R. Immunological memory in humans. Semin. Immunol. 16, 197-203 (2004).

50. Stenger, R.M. et al. Immunodominance in mouse and human CD4 + T-cell responses specific for the Bordetella pertussis virulence factor P.69 pertactin. Infect. Immun. 77, 896-903 (2009).

51. Eijssen, L.M. et al. User-friendly solutions for microarray quality control and pre-processing on ArrayAnalysis.org. Nucleic Acids Res. 41, W71-W76 (2013).

52. Bolstad, B.M., Irizarry, R.A., Astrand, M. \& Speed, T.P. A comparison of normalization methods for high density oligonucleotide array data based on variance and bias. Bioinformatics 19, 185-193 (2003).

53. Dai, M. et al. Evolving gene/transcript definitions significantly alter the interpretation of GeneChip data. Nucleic Acids Res. 33, e175 (2005).

54. Samarajiwa, S.A., Forster, S., Auchettl, K. \& Hertzog, P.J. INTERFEROME: the database of interferon regulated genes. Nucleic Acids Res. 37, D852D857 (2009).

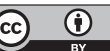

This work is licensed under a Creative Commons Attribution 4.0 International License. Theimages or other third party material in this articleareincluded in thearticle's Creative Commons license, unless indicated otherwise in the credit line; if the material is not included under the Creative Commons license, users will need to obtain permission from the license holder to reproduce the material. To view a copy of this license, visit http://creativecommons.org/licenses/by/4.0/

(C) The Author(s) 2018 